\title{
Investigation of dust and water ice in comet 9P/Tempel 1 from Spitzer observations of the Deep Impact event
}

\author{
A. Gicquel ${ }^{1}$, D. Bockelée-Morvan ${ }^{1}$, V. V. Zakharov ${ }^{1,2}$, M. S. Kelley ${ }^{3}$, C. E. Woodward ${ }^{4}$, and D. H. Wooden ${ }^{5}$ \\ ${ }^{1}$ LESIA, Observatoire de Paris, CNRS, UPMC, Université Paris-Diderot, 5 place Jules Janssen, 92195 Meudon, France \\ e-mail: [adeline.gicquel; dominique.bockelee;vladimir .zakharov]@obspm. fr \\ 2 Gordien Strato, France \\ 3 Department of Astronomy, University of Maryland, College Park, MD 20742-2421, USA \\ e-mail: msk@astro.umd.edu \\ 4 Minnesota Institute for Astrophysics, University of Minnesota, 116 Church St SE, Minneapolis, MN 55455, USA \\ e-mail: chelsea@astro.umn.edu \\ ${ }^{5}$ NASA Ames Research Center, Space Science Division, USA \\ e-mail: dwooden@me.com
}

Received 22 December 2011 / Accepted 9 February 2012

\begin{abstract}
Context. The Spitzer spacecraft monitored the Deep Impact event on 2005 July 4 providing unique infrared spectrophotometric data that enabled exploration of comet 9P/Tempel 1's activity and coma properties prior to and after the collision of the impactor.

Aims. The time series of spectra take with the Spitzer Infrared Spectrograph (IRS) show fluorescence emission of the $\mathrm{H}_{2} \mathrm{O} v_{2}$ band at $6.4 \mu \mathrm{m}$ superimposed on the dust thermal continuum. These data provide constraints on the properties of the dust ejecta cloud (dust size distribution, velocity, and mass), as well as on the water component (origin and mass). Our goal is to determine the dust-to-ice ratio of the material ejected from the impact site.

Methods. The temporal evolution of the continuum was analyzed using a dust thermal model which considers amorphous carbon and intimate silicate-carbon mixtures. The water emission was extracted from the spectra and the water columns within the Spitzer extraction aperture were inferred using a fluorescence excitation model. Time-dependent models simulating the development of the ejecta cloud and the sublimation of icy grains were developed to interpret the temporal evolution of both dust and water emissions within the field of view.

Results. Both the color temperature of the grains in the ejecta cloud $(375 \pm 5 \mathrm{~K})$ and their size distribution show that a large quantity of submicron grains were ejected by the impact. The velocity of the smallest grains is $230 \mathrm{~m} \mathrm{~s}^{-1}$, with a power index for the size dependence of $0.3-0.5$, in agreement with gas loading. The total mass of dust is $(0.7-1.2) \times 10^{5} \mathrm{~kg}$ for grain sizes $0.1-1 \mu \mathrm{m}$ and $(0.5-2.1) \times 10^{6} \mathrm{~kg}$ for sizes $0.1-100 \mu \mathrm{m}$. A sustained production of water is observed, which can be explained by the sublimation of pure ice grains with sizes less than $1 \mu \mathrm{m}$ and comprising a mass of ice of $(0.8-1.8) \times 10^{7} \mathrm{~kg}$. The contribution of dirty ice grains to the ice budget is negligible. Assuming that water was essentially produced by icy grains present in the ejecta cloud, our measurements suggest a very high ice-to-dust ratio of about 10 in the excavated material, which greatly exceeds the gas-to-dust production rate ratio of $\sim 0.5$ measured for the background coma. Alternately, if a large amount of material felt back to the surface and sublimated, ice-to-dust ratios of 1-3 are not excluded. A better understanding of the cratering event on 9P/Tempel 1 is required to disentangle between the two hypotheses. Evidence for grain fragmentation in the ejecta cloud is found in the data. The pre-impact water production rate is measured to be $(4.7 \pm 0.7) \times 10^{27} \mathrm{~mol} \mathrm{~s}^{-1}$.
\end{abstract}

Key words. comets: individual: comet 9P/Tempel 1 - methods: data analysis - methods: numerical - infrared: general

\section{Introduction}

Comets contain the most primitive icy material remaining from the epoch of solar system formation. Comets belonging to Jupiter's dynamical family (Jupiter-family comets; JFCs) have shorter orbital periods than Oort cloud comets and their surfaces could be highly processed by their frequent perihelion passages and higher insolation. The upper layers of JFC cometary nuclei are likely evolved (e.g., Davidsson et al. 2009; A'Hearn 2008; Kelley \& Wooden 2009) and probably have lost a significant fraction of their primodial volatiles.

The NASA Deep Impact (DI) mission to the JFC 9P/Tempel 1 provided the first opportunity to examine material from the interior of a cometary nucleus resulting from a planned collision (A'Hearn et al. 2005). The impact event was observed with ground-based (Meech et al. 2005) and space-based (Keller et al. 2005; Lisse et al. 2006) instrumentation. The DI spacecraft released a $370 \mathrm{~kg}$ impactor that impacted the comet nucleus at $10.3 \mathrm{~km} \mathrm{~s}^{-1}$. This impact produced a large cloud of dust, ice, and volatiles. The principal objective of the DI experiment was to compare the composition of dust and volatiles before and after the impact event and to characterize the amount of material excavated by the collision.

The Spitzer Space Telescope (Werner et al. 2004; Gehrz et al. 2007) obtained a unique infrared spectrophotometic data set enabling exploration of the comet 9P/Tempel 1's activity and coma properties prior to and after the DI event. Here, we discuss new analysis of Spitzer Infrared Spectrograph (IRS; Houck et al. 2004) time series measurements of the 5 to $14 \mu \mathrm{m}$ spectral energy distribution (SED) of the DI enhanced coma. In particular, 
Table 1. Spitzer observational campaign summary.

\begin{tabular}{|c|c|c|c|c|c|c|}
\hline $\begin{array}{l}\text { Time from impact } \\
\text { (h) }\end{array}$ & AOR key & UT date & $\begin{array}{c}r_{\mathrm{h}} \\
(\mathrm{AU})\end{array}$ & $\begin{array}{c}\Delta \\
(\mathrm{AU})\end{array}$ & $\begin{array}{c}\text { Phase angle } \\
\left(^{\circ}\right)\end{array}$ & $\begin{array}{l}\text { Aperture } \\
(\mathrm{km})\end{array}$ \\
\hline-41.3 & 15299072 & $2005-07-02$ 12:52:13 & 1.506 & 0.711 & 36 & $4780 \times 4780$ \\
\hline-22.4 & 15299328 & 2005-07-03 07:25:07 & 1.506 & 0.715 & 36 & $4810 \times 4810$ \\
\hline 0.67 & 15299584 & 2005-07-04 06:30:45 & 1.506 & 0.719 & 36 & $4840 \times 4840$ \\
\hline 1.83 & 15299840 & 2005-07-04 07:50:13 & 1.506 & 0.719 & 36 & $4840 \times 4840$ \\
\hline 3.6 & 15300096 & 2005-07-04 09:24:29 & 1.506 & 0.720 & 36 & $4845 \times 4845$ \\
\hline 4.2 & 15300352 & 2005-07-04 10:01:14 & 1.506 & 0.720 & 36 & $4845 \times 4845$ \\
\hline 10.2 & 15300608 & 2005-07-04 16:02:19 & 1.506 & 0.721 & 36 & $4850 \times 4850$ \\
\hline 20.5 & 15300864 & 2005-07-05 02:19:54 & 1.506 & 0.723 & 36 & $4860 \times 4860$ \\
\hline 41.7 & 15301120 & 2005-07-05 23:34:21 & 1.506 & 0.727 & 37 & $4890 \times 4890$ \\
\hline 122 & 15301376 & 2005-07-09 07:38:14 & 1.507 & 0.743 & 37 & $5000 \times 5000$ \\
\hline 164 & 15301632 & 2005-07-11 01:58:44 & 1.507 & 0.752 & 38 & $5060 \times 5060$ \\
\hline 242 & 15301888 & 2005-07-14 08:14:21 & 1.509 & 0.770 & 38 & $5180 \times 5180$ \\
\hline 740 & 15302144 & 2005-08-04 01:43:10 & 1.536 & 0.901 & 40 & $6060 \times 6060$ \\
\hline 1027 & 15302400 & 2005-08-16 00:29:26 & 1.563 & 0.992 & 40 & $6670 \times 6670$ \\
\hline
\end{tabular}

we examine the evolution of the fluorescence emission of the $v_{2}$ vibrational band of water (isolated by subtracting the observed continuum) and discuss our grain thermal model that probes the properties of the dust in the coma. The data before and after the impact event enable us to study the temporal evolution of dust grains and water molecules. A Previous analysis of those data made by Lisse et al. (2006) focusses on the composition of the dust. Emission signatures due to amorphous and crystalline silicates, amorphous carbon, phyllosilicates, polycyclic aromatic hydrocarbons, water ice, and sulfides were found.

\section{Observations}

Spectral maps covering approximately $20^{\prime \prime} \times 60^{\prime \prime}$ were acquired with the Infrared Spectrograph (IRS) instrument (Houck et al. 2004) on the Spitzer Space Telescope at 14 times from impact $(\mathrm{TI})$ ranging from $\mathrm{TI}-41.3$ to $\mathrm{TI}+1027 \mathrm{~h}$ (Lisse et al. 2006). The impact occurred on 2005 July 04 at 05:44:36 UT (A'Hearn et al. 2005), which corresponds to 05:50:34 UT as seen by Spitzer. The time of observation, Spitzer AOR (Astronomical Observation Request) numbers, heliocentric distance $\left(r_{\mathrm{h}}\right)$, geocentric distance $(\Delta)$ and phase angle are listed in Table 1 . We downloaded the observations as basic calibrated data (BCD) files from the Spitzer archive. The archive contained observations from both the short wavelength, low spectral resolution (SL) and the long wavelength, low spectral resolution (LL) modules of the IRS instrument. However, for the purposes of this paper we only consider the SL module because of the different observing circumstances (orientation, pixel scale, slit width, time from impact) of the two spectral modules. After co-adding all frames, the effective on-source integration time is $58.7 \mathrm{~s}$, and the entire SL spectral map takes $2600 \mathrm{~s}$ to complete.

The SL module is composed of two slits that simultaneously observe independent portions of the sky: SL1, which observes at $7.5-14 \mu \mathrm{m}$ in the first spectral order; and SL2, which observes at 5.2-7.6 $\mu \mathrm{m}$ in the second spectral order. Both slits are imaged on the same $128 \times 128$ pixel array and have approximately the same dimensions $\left(3.6^{\prime \prime} \times 57^{\prime \prime}\right.$ for SL2, and 3.7" $\times$ $57^{\prime \prime}$ for SL1). The imaging array also covers a small region of the first order spectrum that passes through the SL2 slit, providing an additional spectrum from 7.3-8.7 $\mu \mathrm{m}$, often referred to as the "bonus order" or SL3. When one slit is centered on the comet, the other slit is observing blank sky.

The spectral maps are composed of a $2 \times 11$ grid of slit positions (parallel $\times$ perpendicular to the long dimension of the slit), with a step size of $1.8^{\prime \prime}$ in both dimensions. The twodimensional (2D) spectra at each position are background subtracted using the blank sky observations described above. The 44 spectra ( 22 positions $\times 2$ cycles per position) at each epoch are assembled into 3 data cubes (one for SL1, SL2, and SL3) with the CUBISM software version 1.7 (Smith et al. 2007). The data cubes are iteratively inspected (both automatically and manually) for bad pixels and cosmic rays, and re-assembled ignoring those data points. Since IRS spectra are calibrated with observations of point sources, the CUBISM software handles both the necessary aperture and extended source corrections appropriate for observations of spatially resolved comets (Spitzer Science Center 2011). Flux uncertainties are propagated from the original BCD files into separate spectral data cubes. We extract our spectra from the final data cubes in $9.25^{\prime \prime} \times 9.25^{\prime \prime}$ $(5 \times 5$ pixels $)$ apertures. The individual orders are scaled to produce a continuous spectrum, holding the flux of the SL1 order fixed. Most scale factors correspond to less than a $10 \%$ correction, but occasionally corrections up to $20 \%$ were necessary to match SL3 with SL1. We identify two likely sources for the scale factors. First, the intrinsic calibration uncertainty of the IRS is quoted to be $<5 \%$ (IRS Instrument Handbook). Second, large order mismatches can arise from pointing offsets. Pointing errors, ephemeris errors, and the rapidly moving ejecta could introduce an offset between the SL1 and SL2/3 spectral maps. Moreover, the CUBISM interpolation from a $2 \mathrm{D}$ to a $3 \mathrm{D}$ data set could introduce additional offsets between the two spectral maps.

Although the SL1 order covers wavelengths up to $\approx 14 \mu \mathrm{m}$, the spectra are not reliable at beyond $\approx 13.5 \mu \mathrm{m}$ due to the data artifact known as the "teardrop". The teardrop is a region of excess flux that may be caused by reflections internal to the instrument (Spitzer Science Center, 2011). The teardrop shows up spatially as excess emission along one side of a source. It is easily identifiable in the 2D spectra of point sources (e.g., calibration stars) since they are approximately spatially symmetric, but more difficult to identify in our 2D spectra of comet 9P/Tempel 1 . However, the teardrop does appear to be present in our spectra, and we ignore all data at wavelengths $\lambda>13.2 \mu \mathrm{m}$.

Thermal emission from the nucleus contributes to a significant fraction of the spectral flux in the $9.25^{\prime \prime} \times 9.25^{\prime \prime}$ onnucleus apertures, ranging from 20 to $50 \%$ of the flux in preimpact spectra (the exact value depends on wavelength), and 10 to $20 \%$ of the flux in the first post-impact spectrum. We use the near-Earth asteroid thermal model, (NEATM; Harris 1998), with the effective radius $(3.3 \mathrm{~km})$ and IR-beaming parameter $(\eta=0.9)$ 


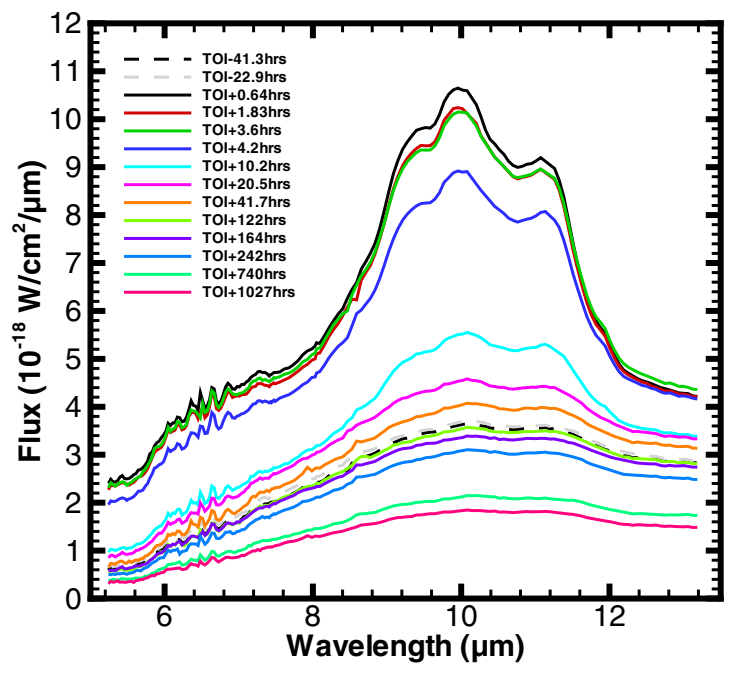

Fig. 1. Spitzer SL1, SL2 and SL3 spectra of comet 9P/Tempel 1 at different times from impact for a FOV of 9.25" $\times 9.25^{\prime \prime}$ (corresponding to an area of $\sim 4900 \times 4900 \mathrm{~km}$ within the coma) centered on the nucleus. The contribution of the nucleus was removed from all spectra.

as measured in Spitzer observations of the nucleus at $r_{\mathrm{h}}=3.7 \mathrm{AU}$ (Lisse et al. 2005). The computed NEATM spectrum is corrected for wavelength dependent aperture losses, considering the square aperture size, point spread function of the instrument (computed with Tiny Tim/Spitzer), and assuming the nucleus is located at the ephemeris position of the comet. The contribution of the nucleus was removed from all spectra.

The spectra display continuum emission due to dust (silicates and carbonaceous grains) and additional emission from water (including the $v_{2}$ band at $6.4 \mu \mathrm{m}$; Woodward et al. 2007; Bockelée-Morvan et al. 2009). This set of observations enables us to probe the temporal variation in the dust grain properties (with the SL1, SL2, and SL3 modules) and the number of water molecules (with the SL2 module) in the field of view (FOV). Spectra acquired with the SL1, SL2 and SL3 modules are shown in Fig. 1.

\section{Analysis of dust thermal emission}

The temporal evolution and shape of the dust spectra as observed by Spitzer provides constraints on the size distribution of dust grains after impact.

The continuum color temperature of the coma dust, $T_{\text {color }}$, is determined by fitting the dust continuum emission between 5.2-5.8 $\mu \mathrm{m}, 7.4-7.6 \mu \mathrm{m}$, and $12.4-13.2 \mu \mathrm{m}$ with a blackbody. The 5.8-7.4 $\mu \mathrm{m}$ and 7.6-12.4 $\mu \mathrm{m}$ spectral regions show spectral signatures from water and silicates, respectively. All continua in SL1, SL2 and SL3 spectra are used to establish the best constraint on the temperature.

The dust color temperature before impact is $265 \pm 2 \mathrm{~K}$. $T_{\text {color }}$ within the FOV increases to $315 \pm 5 \mathrm{~K}$ just after impact (Fig. 2). At TI $+41.7 \mathrm{~h}, T_{\text {color }}$ of the coma dust returns to the value before impact. To determine $T_{\text {color }}$ of the ejecta lifted into the coma as a result of the DI event, the spectrum obtained at $\mathrm{TI}-41.3 \mathrm{~h}$ was subtracted from the post-impact spectra. Figure 3 shows selected spectra of the ejecta and fitted blackbodies. We find that $T_{\text {color }}$ of the ejecta, $\simeq 375 \pm 5 \mathrm{~K}$, is consistent with $T_{\text {color }}=390 \mathrm{~K}$ determined by Lisse et al. (2007) from the same data set. $T_{\text {color }}$ of the ejecta is higher than that of the background coma. To first order, this temperature difference can be explained solely by a change in the grain size

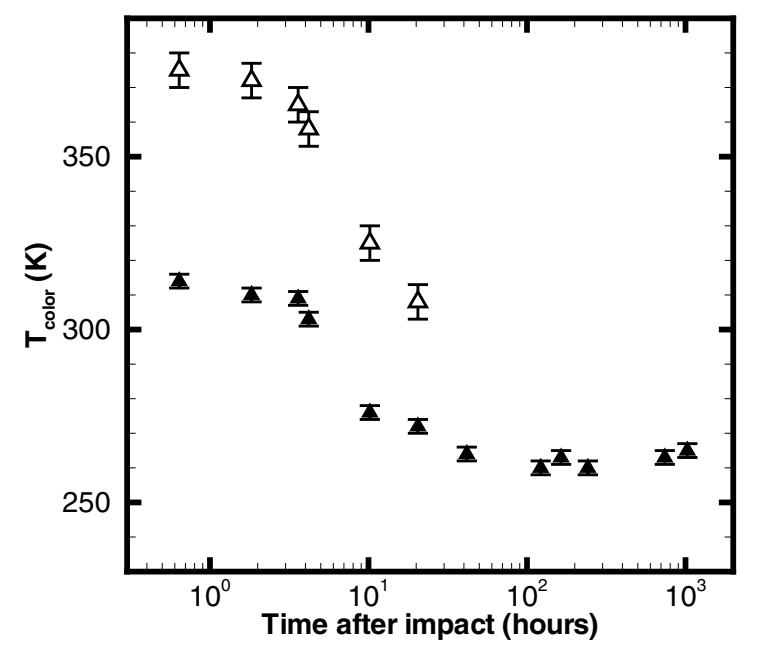

Fig. 2. Temporal evolution of the color temperature, $T_{\text {color }}$, of the overall dust (filled triangles), and of the ejecta (open triangles) within a $9.25^{\prime \prime} \times$ 9.25" FOV centered on the nucleus.

dominating the coma. As small grains are normally hotter than large grains (Fig. 4), the higher $T_{\text {color }}$ suggests that the grains ejected by the impact were significant number of grains smaller than in the background coma. Our assertion is corroborated by other analyses of the DI induced coma (Sugita et al. 2005; Keller et al. 2005; Lisse et al. 2006; Sunshine et al. 2006; Harker et al. 2007).

\subsection{Model of dust grains}

Model thermal emission spectra were constructed for amorphous olivine, amorphous pyroxene, and amorphous carbon dust grains. Our analytical description is similar to techniques used by Lisse et al. (1998), Hanner et al. (1985), Harker et al. (2002), and Harker et al. (2007) to model dust thermal emission from comet grains in an optically-thin coma.

To compute the temperature of the dust grains in the coma, it is assumed that the energy received from the Sun and reradiated in the infrared are in radiative equilibrium. The major source of heating for a dust grain is the absorption of solar radiation (Lamy 1974):

$E_{\text {sun }}=\pi\left(\frac{r_{\text {Sun }}}{r_{\mathrm{h}}}\right)^{2} \pi a^{2} \int_{0}^{\infty} Q_{\mathrm{a}}(a, \lambda) B_{\lambda}\left(T_{\text {Sun }}, \lambda\right) \mathrm{d} \lambda$

where $a$ is the grain radius, $r_{\text {Sun }}$ is the radius of the Sun and $r_{\mathrm{h}}$ is the heliocentric distance, $B_{\lambda}$ is the Planck radiation function, $T_{\text {Sun }}=5770 \mathrm{~K}$ is the Sun temperature, $Q_{\mathrm{a}}$ is the absorption efficiency of the grain and $\lambda$ is the wavelength. We assumed that $Q_{\mathrm{a}}=Q_{\mathrm{e}}$ according to the Kirchhoff's law, where $Q_{\mathrm{e}}$ is the emission efficiency.

We assumed that dust grains are cooling via thermal radiation:

$E_{\mathrm{rad}}=4 \pi a^{2} \int_{0}^{\infty} Q_{\mathrm{a}}(a, \lambda) \pi B_{\lambda}\left(T_{\mathrm{d}}, \lambda\right) \mathrm{d} \lambda$

where $T_{\mathrm{d}}$ is the equilibrium temperature of the grain. Following Lisse et al. (1998) and Lien (1990) we assumed that other heating and cooling processes (i.e., sublimation) are negligible. 

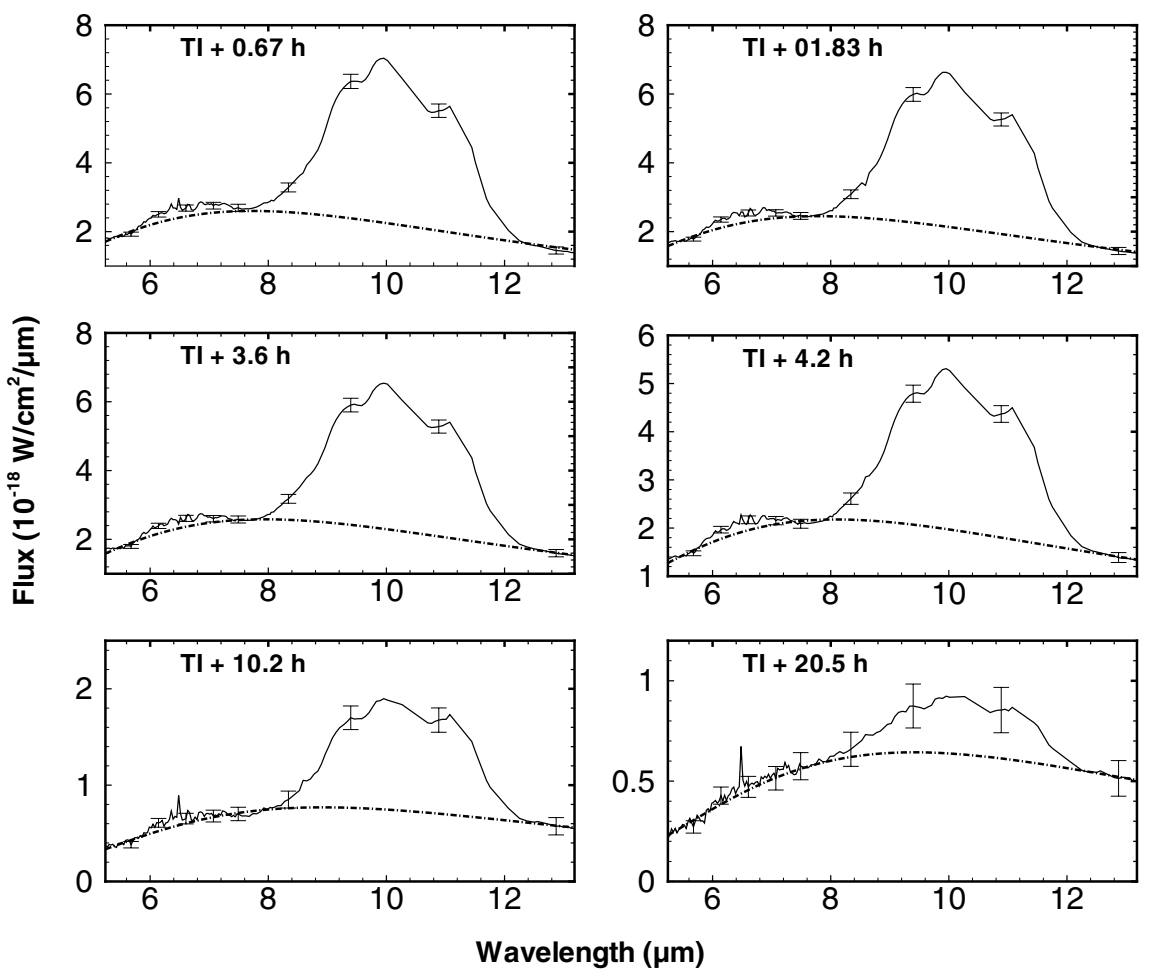

Fig. 3. Spitzer spectra of the ejectas from comet 9P/Tempel 1 at different times after DI event. Grey dashed lines show the fitted blackbody radiation from which dust color temperatures $T_{\text {color }}=375 \pm 5 \mathrm{~K}, 372 \pm 5 \mathrm{~K}, 365 \pm 5 \mathrm{~K}$, $358 \pm 5 \mathrm{~K}, 325 \pm 5 \mathrm{~K}, 308 \pm 5 \mathrm{~K}$ were derived at TI $+0.67 \mathrm{~h}, \mathrm{TI}+1.83 \mathrm{~h}, \mathrm{TI}+3.6 \mathrm{~h}, \mathrm{TI}+4.2 \mathrm{~h}$, $\mathrm{TI}+10.2 \mathrm{~h}$, and TI $+20.5 \mathrm{~h}$, respectively.

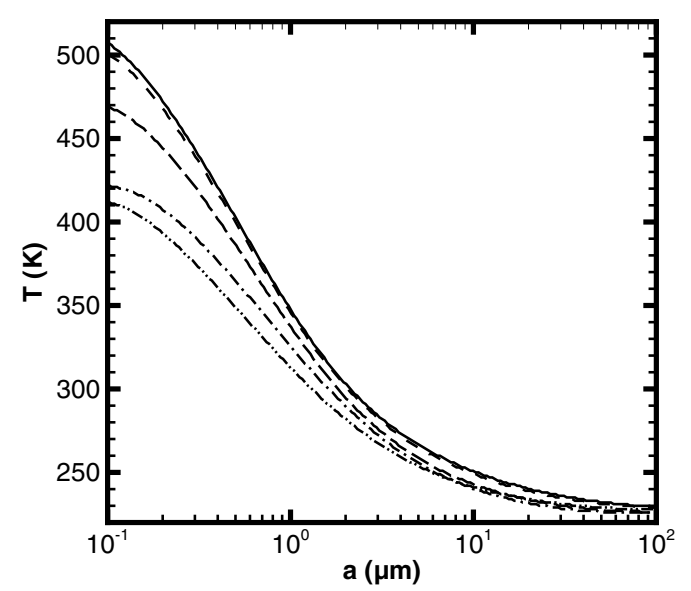

Fig. 4. The equilibrium temperature of porous grains $(D=2.727)$ at $r_{\mathrm{h}}=$ 1.506 AU: amorphous carbon (solid line), amorphous olivine (dashdotted-dotted line), two-layer grains with $\alpha=0.9$ (dashed line), with $\alpha=0.5$ (long-dashed line) and with $\alpha=0.1$ (dash-dotted line).

The observed flux from thermal emission produced by a collection of grains of various radii is then:

$F_{\lambda}=\int_{a_{\min }}^{a_{\max }} \frac{\pi a^{2}}{\Delta^{2}} Q_{\mathrm{a}}(a, \lambda) B_{\lambda}\left(T_{\mathrm{d}}(a), \lambda\right) n(a) \mathrm{d} a$

where $n(a)$ is the size distribution of grains in the FOV. The resultant model spectrum is compared with the observations and the particle size distribution is adjusted to provide the minimum $\chi^{2}$.

For the grain size distribution we used two different formulae:

1. the power law distribution:

$n(a)=\Gamma a^{-q}$,

and
2. the Hanner modified power law (Hanner et al. 1985), which provides an adequate model for infrared comet data ranging form 3.05 to $20 \mu \mathrm{m}$ :

$n(a)=\Gamma\left(1-\frac{a_{0}}{a}\right)^{M_{\mathrm{h}}}\left(\frac{a_{0}}{a}\right)^{N_{\mathrm{h}}}$.

In these equations, $\Gamma$ is a normalization constant. In the power law distribution $q$ determines the slope of the distribution. In the Hanner distribution $a_{0}$ is the minimum grain radius, $N_{\mathrm{h}}$ determines the slope of the distribution at large $a$, and $M_{\mathrm{h}}$ is related to the radius of the peak of the size distribution $a_{\mathrm{p}}$ by:

$a_{\mathrm{p}}=a_{0} \frac{\left(M_{\mathrm{h}}+N_{\mathrm{h}}\right)}{N_{\mathrm{h}}}$.

Hanner (1984) argues that $3.7 \leq N_{\mathrm{h}} \leq 4.2$ is typical in cometary atmospheres. Following Harker et al. (2007), we adopt a value of $N_{\mathrm{h}}=3.7$. The value of $N_{\mathrm{h}}$ is best constrained when fitting spectra with a broad wavelength range that spans the peak thermal emission.

The intrinsic properties of a dust grain are contained within $Q_{\mathrm{a}}$ : grain size, shape and composition (including mineralogy and porosity). We used Mie theory (Bohren \& Huffman 1983; Van de Hulst 1957), which is the most common technique used throughout the literature (Lisse et al. 1998; Hanner et al. 1996, 1994; Harker et al. 2002), to compute $Q_{\mathrm{a}}$ for spherical grains of given radius and wavelength-dependent indices of refraction, $n$ and $k$. Mie theory can be used to compute the absorption efficiency in the case where $Q_{\mathrm{a}}$ varies slowly with wavelength, for example amorphous grains. However Mie theory cannot be applied to calculations for an emission feature where the optical constants are rapidly varying, e.g., crystalline grains (Kolokolova et al. 2004).

Three grain compositions are considered: amorphous silicates (olivine or pyroxene with fractional proportion $\mathrm{Mg}=\mathrm{Fe}=0.5$; Harker et al. 2007), amorphous carbon, and two-layer grains. The two-layer composition is based on the 
structure of cometary grains outlined by Greenberg \& Hage (1990). The basic building block consists of a silicate core surrounded by an organic component. We neglected water ice grains in our thermal model as explained in Sect. 4.4. Since our goal is to describe the continuum and not to fit in detail the shape of the silicate feature, we ignored crystalline silicates.

The indices of refraction that we used to calculate $Q_{\mathrm{a}}$ for the amorphous silicates are from Dorschner et al. (1995), and for the amorphous carbon from Edoh (1983). For the two-layer grains, the Maxwell Garnett effective medium theory was used to calculate the effective refractive index $m_{b i}$ of a core-mantle composite particle (Greenberg \& Hage 1990):

$$
m_{b i}^{2}=m_{\mathrm{m}}^{2}\left\{1+3 q_{b i}^{3} \frac{\frac{m_{\mathrm{c}}^{2}-m_{\mathrm{m}}^{2}}{m_{\mathrm{c}}^{2}+2 m_{\mathrm{m}}^{2}}}{1-q_{b i}^{3}\left(\frac{m_{\mathrm{c}}^{2}-m_{\mathrm{m}}^{2}}{m_{\mathrm{c}}^{2}+2 m_{\mathrm{m}}^{2}}\right)}\right\}
$$

where $m_{\mathrm{m}}$ and $m_{\mathrm{c}}$ are respectively the refractive indices of the mantle component and the core component and $q_{b i}$ is the fractional radius which depends of the fractional mass of the mantle component:

$\alpha=M_{\mathrm{m}} /\left(M_{\mathrm{c}}+M_{\mathrm{m}}\right)$

where $M_{\mathrm{m}}$ and $M_{\mathrm{c}}$ are respectively the mass of the mantle and of the core. We adopt a density of $\rho_{\mathrm{C}}=2.5 \mathrm{~g} \mathrm{~cm}^{-3}$ for carbon grains and of $\rho_{\mathrm{Si}}=3.3 \mathrm{~g} \mathrm{~cm}^{-3}$ for silicate grains (Harker et al. 2002).

We assumed that grains are porous and we used the fractal model introduced by Lisse et al. (1998) for the porosity:

$p=1-\left(a / a_{0}\right)^{D-3}$

where $p$ is fraction of vacuum and $D=3.0$ corresponding to solid grains. We set $D=2.727$ which is the best fit fractal dimension for 9P/Tempel's ejecta following Harker et al. (2007).

The refractive index $m_{\mathrm{p}}$ of porous grain aggregates was calculated using a form of the Maxwell Garnett formula:

$m_{\mathrm{p}}^{2}=1+\frac{3(1-p)\left(m_{\mathrm{s}}^{2}-1\right) /\left(m_{\mathrm{s}}^{2}+2\right)}{1-(1-p)\left(m_{\mathrm{s}}^{2}-1\right) /\left(m_{\mathrm{s}}^{2}+2\right)}$

where $m_{\mathrm{s}}$ is the refractive index of the solid material.

The equilibrium temperatures of the different grain compositions is shown in Fig. 4. The temperature of carbon grains is higher than the temperature of silicate grains, and the temperature of the two-layer grains lies between the two.

Using a $\chi^{2}$ minimisation method, the best fit for the dust continuum of the ejecta is determined for each composition of grain for each time stamp, where the observed SL1, SL2 and SL3 data are used to constrain the model parameters. Our method differs from the approach of Harker et al. (2007) who constrain the size distribution by fitting simultaneously the continuum and the shape of the silicate signatures.

\subsection{Temporal evolution of grain size distribution}

To assess the temporal evolution of the coma grain size distribution of comet 9P/Tempel 1, model spectra are computed by integrating over a grain size range from $a_{\min }$ to $a_{\max }$. The value $a_{\max }=100 \mu \mathrm{m}$ is fixed but $a_{\min } \geq a_{0}=0.1 \mu \mathrm{m}$ is variable. In order to reproduce the number of free parameters, we set $a_{\min }=0.1 \mu \mathrm{m}$ for all grain compositions and size distribution, except when using amorphous carbon with the power law size distribution (Eq. (4)). The exact value of $a_{\max }$ has a minimal impact on the determination of the grain size distribution because the large grains are too cold and do not contribute significantly to the observed flux at wavelengths $<20 \mu \mathrm{m}$ (Harker et al. 2002). In addition, amorphous silicates are colder than amorphous carbon and therefore do not dominate the continuum near $10 \mu \mathrm{m}$, so we only need to use two grain compositions: amorphous carbon and two-layer grains. We have then two free parameters when using the power-law size distribution: $a_{\min }$ and $q$ for the amorphous carbon case, and $\alpha$ and $q$ for the twolayer grains. As for the Hanner size distribution, we set the slope to be $N_{\mathrm{h}}=3.7$, so the free parameters are: $a_{\mathrm{p}}$ for the amorphous carbon case, and $\alpha$ and $a_{\mathrm{p}}$ for the two-layer grains.

The two-layer grain model that best fits the wing of the $10 \mu \mathrm{m}$ emission feature from amorphous silicates (Fig. 5) at $\mathrm{TI}+0.67 \mathrm{~h}$ corresponds to a core of amorphous olivine covered by a mantle of amorphous carbon with a fractional mass for the mantle $\alpha=0.1$. We then fixed the silicate/carbon ratio to this value for all other post-impact spectra ( $\alpha$ is not a free parameter now), neglecting amorphous pyroxene. This fractional mass $\alpha=0.1$ is smaller than the fractional mass derived by Harker et al. (2007) of $\sim 0.35$. Our thermal model uses amorphous carbon grains and/or two-layer grains and thus differs from Harker et al. (2007) who used a mixing of independent grains composed of amorphous carbon and amorphous or crystalline silicates. In the analysis of Harker et al. (2007), the silicate/carbon ratio is a free parameter which increases with time after impact, and the increase of the $10 \mu \mathrm{m}$ silicate feature-to-continuum is associated with the production of submicron size silicate grains. We have a different model outcome wherein the silicate/carbon ratio remains constant and the $10 \mu \mathrm{m}$ silicate feature-to-continuum is the same for each time after impact. At TI $+20.5 \mathrm{~h}$, the $10 \mu \mathrm{m}$ silicate emission feature due to the ejecta has a smaller contrast with respect to the continuum, because of the decrease of the silicate/carbon ratio according to Harker et al. (2007), subsequently our model does not accurately reproduce the observed SED (Fig. 5). For the two-layer grains, since $\alpha$ is no longer a free parameter and $a_{\text {min }}$ is fixed, the minimum $\chi^{2}$ is determined by adjusting only $q$, when considering the power law distribution (Eq. (4)), and by adjusting only $a_{\mathrm{p}}$ when considering the Hanner distribution (Eq. (5)). We used a confidence level of $68.3 \%$ to determine the uncertainty on the parameters of the size distribution.

Before impact, we find that the coefficients for the powerlaw distribution are $a_{\min }=1.08 \pm 0.2 \mu \mathrm{m}$ and $q=2.33 \pm 0.1$ in the case of amorphous carbon grains while $q=2.62 \pm 0.1$ for the two-layer grains. For the Hanner distribution, we find $a_{\mathrm{p}}=2.98 \pm 0.05 \mu \mathrm{m}$ for the amorphous carbon grain case, while $a_{\mathrm{p}}=1.1 \pm 0.1 \mu \mathrm{m}$ when two-layer grains are used. Figure 6 presents the temporal evolution of the parameters $a_{\mathrm{p}}, a_{\min }$ and $q$ after impact. The $\chi_{v}^{2}$ of the best fits, utilizing 36 data points, are in the range $0.13-1.13$, and $0.12-1.15$ for the power law and Hanner distributions, respectively.

In the case of the Hanner distribution (Fig. 6b), $a_{\mathrm{p}}$ after impact is smaller than before impact while increasing with time post-impact. The derived $a_{\mathrm{p}}$ value just after impact $(\mathrm{TI}+0.67 \mathrm{~h})$ is $0.42 \pm 0.01 \mu \mathrm{m}$ for the amorphous carbon. This value is two times higher than that obtained by Harker et al. (2007) from the analysis of the $10-\mu \mathrm{m}$ emission feature. One reason for this variance could be that the submicron sized grains contributing to the continuum emission are cooler than pure amorphous carbon grains. Carbonsilicate mixtures are cooler than amorphous carbon grains (Fig. 4) and are expected to be more representative of comet 

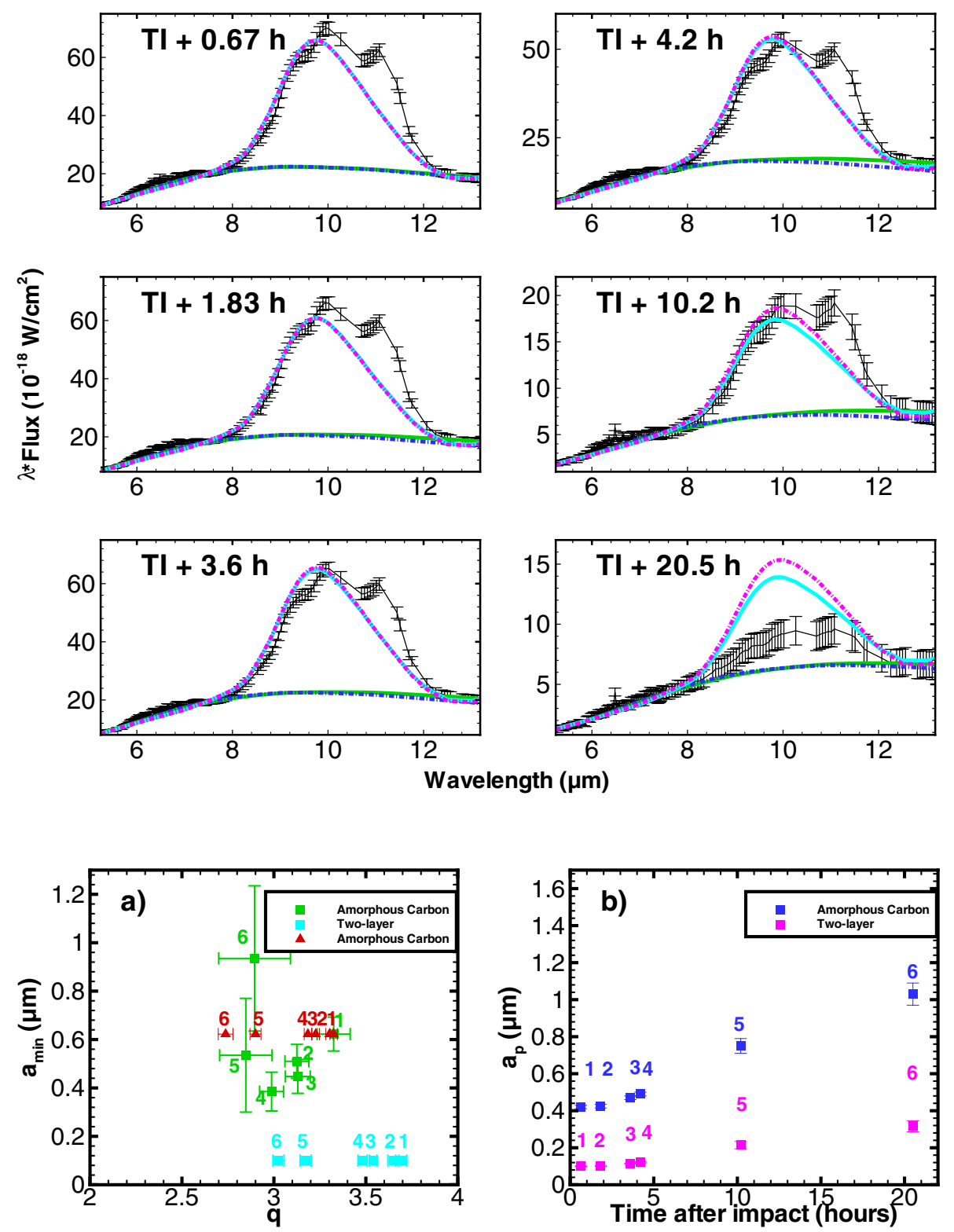

Fig. 6. Derived grain parameters a) for the power law distribution and $\mathbf{b}$ ) for the Hanner distribution from fits to the Spitzer ejecta spectra of comet 9P/Tempel 1 . In the left figure, the cyan squares and red triangles correspond to results obtained by fixing $a_{\min }$ to 0.1 and $0.6 \mu \mathrm{m}$, respectively. The different times are labelled according to: (1) TI $+0.67 \mathrm{~h}$; (2) $\mathrm{TI}+1.83 \mathrm{~h}$; (3) $\mathrm{TI}+3.6 \mathrm{~h}$; (4) $\mathrm{TI}+4.2 \mathrm{~h}$; (5) $\mathrm{TI}+10.2 \mathrm{~h}$; (6) $\mathrm{TI}+20.5 \mathrm{~h}$. dust (e.g., Brownlee et al. 2009; Hanner \& Zolensky 2010). As shown in Fig. $6 \mathrm{~b}$, the derived $a_{\mathrm{p}}$ value for the two-layer grains is $0.1 \mu \mathrm{m}$, and smaller than the value obtained by Harker et al. (2007), which might suggest that the thermal properties of the dust contributing to the continuum are intermediate between the amorphous and two-layer cases considered in our work. Given the different approaches used in the two studies, we consider the agreement satisfactory. The value $a_{\mathrm{p}}=0.1 \mu \mathrm{m}$ determined for the two-layer grain composition just after impact corresponds to $M_{\mathrm{h}}=0$ in the Hanner law. The Hanner distribution is then equivalent to a power-law size distribution with a slope index equal to 3.7. Interestingly, the slope of the power-law distribution inferred just after impact is near 3.7. The peak in the Hanner grain size distribution, $a_{\mathrm{p}}$, increases with time (Fig. 6b) as small grains (which have a high velocity) escape from the FOV faster than large grains (see Sect. 3.4).

The interpretation of the time dependence of the minimum grain size in the power-law distribution (Fig. 6a) is not obvious. This free parameter (for carbon grains) is found to decrease with time up to TI $+4.2 \mathrm{~h}$. This apparent variation of the grain size cut-off with time could be explained by the presence in the
FOV of another material which is able to modify the optical properties of the grain distribution, or by the fragmentation of grains. Whatever the material composition (amorphous carbon or two-layer, Fig. 6a), the $q$ index is decreasing with time, indicating the escape of small grains from the FOV. The large uncertainty of $q$ at TI +10.2 and $\mathrm{TI}+20.5 \mathrm{~h}$ arises from the low signal-to noise ratio of the ejecta spectra, and no physical interpretation regarding the temporal variation of $q$ at these epochs is possible.

\subsection{Temporal evolution of the mass of the ejecta}

The parameters of the size distribution $\left(a_{\min }, q, a_{\mathrm{p}}\right)$ enable determination of the mass of the ejecta and its temporal evolution (Fig. 7).

If one considers times before $\mathrm{TI}+10.2 \mathrm{~h}$, ejecta dust mass estimates $\left(M_{\mathrm{d}}\right)$ for grains ranging from $a_{\min }$ to $10 \mu \mathrm{m}$ are similar within a factor of $20 \%$, independent of the size distribution and dust composition (Fig. 7). However, the dust masses determined for the $a_{\mathrm{min}}-100 \mu \mathrm{m}$ size range are model dependent, with higher $M_{\mathrm{d}}$ found for amorphous carbon grains with a power-law 
Table 2. Derived ejecta dust masses from the Spitzer IRS data.

\begin{tabular}{|c|c|c|c|c|c|}
\hline Size distribution & Grain composition & $\begin{array}{l}\text { Size range } \\
\quad(\mu \mathrm{m})\end{array}$ & Ratio & $\begin{array}{c}M_{\mathrm{d}} \\
(\mathrm{kg})\end{array}$ & $\begin{array}{l}M_{\mathrm{d}}^{\text {tot }} \\
(\mathrm{kg})\end{array}$ \\
\hline \multirow[t]{2}{*}{ Hanner } & carbon & $\begin{array}{c}0.1-100 \\
0.1-10 \\
0.1-1\end{array}$ & $\mathrm{Si} / \mathrm{C}=4$ & $\begin{array}{l}(4.5 \pm 0.1) \times 10^{5} \\
(1.8 \pm 0.1) \times 10^{5} \\
(1.3 \pm 0.1) \times 10^{4}\end{array}$ & $\begin{array}{l}(2.2 \pm 0.1) \times 10^{6} \\
(9.0 \pm 0.1) \times 10^{5} \\
(6.6 \pm 0.1) \times 10^{4}\end{array}$ \\
\hline & two-layer & $\begin{array}{c}0.1-100 \\
0.1-10 \\
0.1-1\end{array}$ & Crys $/$ Am $=0.3$ & $\begin{array}{l}(3.6 \pm 0.2) \times 10^{5} \\
(2.1 \pm 0.1) \times 10^{5} \\
(9.2 \pm 0.1) \times 10^{4}\end{array}$ & $\begin{array}{l}(4.7 \pm 0.2) \times 10^{5} \\
(2.7 \pm 0.1) \times 10^{5} \\
(1.2 \pm 0.1) \times 10^{5}\end{array}$ \\
\hline \multirow[t]{2}{*}{ Power law } & carbon & $\begin{array}{c}a_{\min }-100 \\
a_{\min }-10 \\
a_{\min }-1\end{array}$ & $\mathrm{Si} / \mathrm{C}=4$ & $\begin{array}{l}(7.2 \pm 0.6) \times 10^{5} \\
(1.6 \pm 0.1) \times 10^{5} \\
(1.6 \pm 0.1) \times 10^{4}\end{array}$ & $\begin{array}{l}(3.6 \pm 0.6) \times 10^{6} \\
(8.0 \pm 0.1) \times 10^{5} \\
(8.1 \pm 0.1) \times 10^{4}\end{array}$ \\
\hline & two-layer & $\begin{array}{c}0.1-100 \\
0.1-10 \\
0.1-1\end{array}$ & Crys $/$ Am $=0.3$ & $\begin{array}{l}(4.3 \pm 0.3) \times 10^{5} \\
(2.2 \pm 0.1) \times 10^{5} \\
(9.2 \pm 0.1) \times 10^{4}\end{array}$ & $\begin{array}{l}(5.6 \pm 0.3) \times 10^{5} \\
(2.9 \pm 0.1) \times 10^{5} \\
(1.2 \pm 0.1) \times 10^{5}\end{array}$ \\
\hline
\end{tabular}

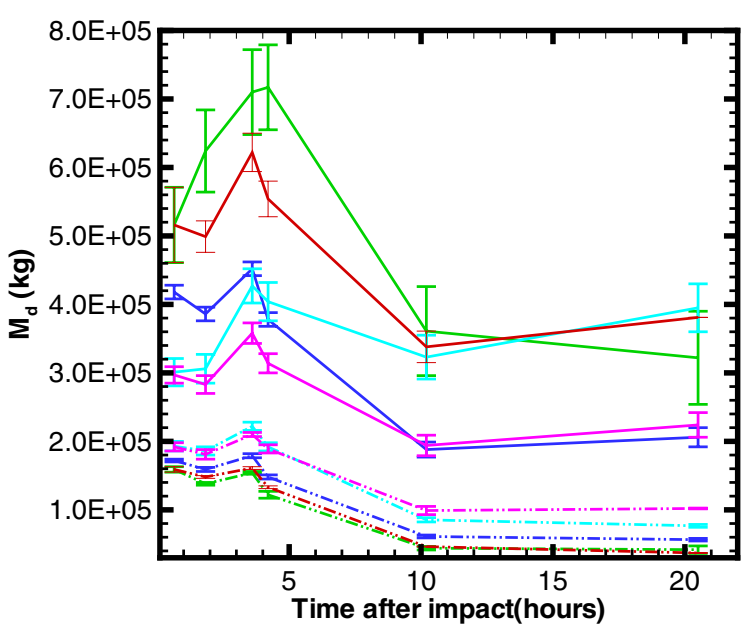

Fig. 7. The mass of ejecta $\left(M_{\mathrm{d}}\right)$ for grain size ranges $a_{\min }-10 \mu \mathrm{m}$ (dash-dotted lines) and $a_{\min }-100 \mu \mathrm{m}$ (solid lines). The different colors correspond to different size distributions and material compositions: green (power-law distribution and amorphous carbon, with $a_{\text {min }}$ as a free parameters), red (power-law distribution and amorphous carbon, with $a_{\min }=0.62 \mu \mathrm{m}$ ), cyan (power-law distribution and two-layer grain), blue (Hanner distribution and amorphous carbon) and purple (Hanner distribution and two-layer grain). The contribution from ice grains is not considered.

distribution. For TI $+10.2 \mathrm{~h}$ and after, mass estimations are consistent for both compositions as the $q$-values of the two-layer and amorphous carbon cases become similar (Fig. 7). The apparently large increase of the carbon mass when $a_{\min }$ is a free parameter is not physically realistic, and artificially arises from variations in the grain size cut-off. Except for this latter happenstance, between TI $+0.67 \mathrm{~h}$ and $\mathrm{TI}+1.83 \mathrm{~h}$, the ejecta mass appears to slightly decrease with time (within the uncertainties). Again this did not physically occur. After TI $+1.83 \mathrm{~h}$, the mass of ejecta is increasing. $M_{\mathrm{d}}$ reaches a maximum at TI $+3.6 \mathrm{~h}$, and decreases after this time, i.e., when small particles start to leave the FOV. For the power-law distribution, $M_{\mathrm{d}}$ increases by $\simeq 20 \%$ between $\mathrm{TI}+1.83 \mathrm{~h}$ and $\mathrm{TI}+3.6 \mathrm{~h}$ even though $q$ changes less than $5 \%$ (Fig. 6). This effect arises because the dust mass is very sensitive to the slope of the power law distribution. The sensitivity increases with the increase of $q$.

We estimate the mass of the ejecta (grains in the range $a_{\min }-100 \mu \mathrm{m}$ ) to be between $3.6 \times 10^{5} \mathrm{~kg}$ and $7.2 \times 10^{5} \mathrm{~kg}$ (Table 2). This mass estimate, based on the measurements at $\mathrm{TI}+4.2 \mathrm{~h}$, does not include crystalline silicates. In addition,
Table 3. Published DI event ejecta dust masses.

\begin{tabular}{lccc}
\hline \hline Study & Telescope & $\begin{array}{c}\text { Size range } \\
(\mu \mathrm{m})\end{array}$ & $\begin{array}{c}M_{\mathrm{d}}^{\text {tot }} \\
(\mathrm{kg})\end{array}$ \\
\hline This work & Spitzer & $0.1-1$ & $(0.7-1.2) \times 10^{5}$ \\
& & $0.1-10$ & $(2.7-8.9) \times 10^{5}$ \\
& & $0.1-100$ & $(0.5-2.1) \times 10^{6}$ \\
Harker et al. (2005) & Gemini-N & $0.1-1$ & $7.3 \times 10^{4}$ \\
& & $0.1-100$ & $1.5 \times 10^{6}$ \\
Keller et al. (2005) & Rosetta & $0.1-500$ & $7.0 \times 10^{6}$ \\
Lisse et al. (2006) & Spitzer & $0.1-10$ & $8.0 \times 10^{5}$ \\
& & $0.1-10^{6}$ & $1.3 \times 10^{6}$ \\
Sugita et al. (2005) & Subaru & $0.1-10$ & $5.0 \times 10^{5}$ \\
& & $0.1-10^{6}$ & $7.0 \times 10^{7}$ \\
Küppers et al. (2005) & Rosetta & $0.1-1$ & $4.4 \times 10^{5}$ \\
Jorda et al. (2007) & Rosetta & $0.1-1.4$ & $(1.3-1.7) \times 10^{5}$ \\
& & $0.1-100$ & $(1.0-14.0) \times 10^{6}$ \\
\hline
\end{tabular}

when only amorphous carbon is considered in the grain model, a correction factor should be applied to account for the contribution of silicates to the total dust mass. Harker et al. (2007) determined a silicate/carbon mass ratio $\mathrm{Si} / \mathrm{C} \sim 4$ for grains between $0.1-1 \mu \mathrm{m}$, with a crystalline/amorphous silicate mass fraction Crys/Am $\sim 0.3$. We assumed this grain composition to calculate the total mass of the ejecta (Table 2). As reported in Table 3, they range from $(2.7-9.0) \times 10^{5} \mathrm{~kg}$ for sizes $0.1-10 \mu \mathrm{m}$, and from $(0.5-2.2) \times 10^{6} \mathrm{~kg}$ for sizes $0.1-100 \mu \mathrm{m}$.

The total amount of dust ejecta released by the Deep Impact experiment is extremely dependent on the size cut-off, varying from $(0.7-4.4) \times 10^{5} \mathrm{~kg}$ for size between $0.1-1 \mu \mathrm{m}$ and from $(0.1-7.0) \times 10^{7} \mathrm{~kg}$ for size between $0.1 \mu \mathrm{m}-1 \mathrm{~m}$ as found in the literature (Table 3). Directly comparing of our model results for $M_{\mathrm{d}}^{\text {tot }}$ to others (Table 3 ) is challenging because of the different analytical methodologies and model assumptions, including size distribution and thermal modeling. However our total mass estimates are in agreement with Harker et al. (2005) for grain sizes ranging from $0.1-1 \mu \mathrm{m}$, with Lisse et al. (2006) and Sugita et al. (2005) for grain sizes ranging from $0.1-10 \mu \mathrm{m}$, and with Harker et al. (2005) and Jorda et al. (2007) for grain sizes ranging from $0.1-100 \mu \mathrm{m}$.

\subsection{Temporal evolution of dust emission and velocity}

We have developed a time-dependent dust model to study the temporal evolution of the dust emission. The amount of dust 


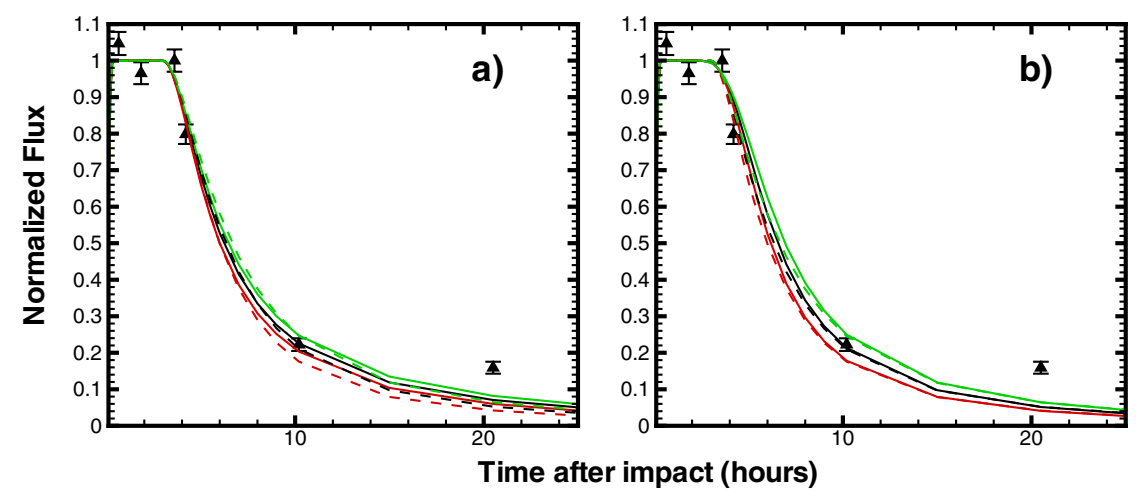

Fig. 8. Model fits to the flux at $5.7 \mu \mathrm{m}$ from ejecta observed in the nucleus-centered $9.25^{\prime \prime} \times$ $9.25^{\prime \prime}$ extraction. Fluxes are normalized to the value measured at TI $+3.6 \mathrm{~h}$. Data are shown with filled symbols with error bars. Results from a time-dependent dust model considering a short-duration (1000 s) outburst are shown both for a power-law size distribution a) and a Hanner distribution b) for amorphous carbon grains (solid line) and two-layer grains (dashed line). Black lines are for the best slope of the velocity distribution $q_{\mathrm{v}}$, green lines are for $q_{\mathrm{v}}+$ 0.05 and red lines are for $q_{\mathrm{v}}-0.05$. The value of $v_{\text {ref }}=230 \mathrm{~m} \mathrm{~s}^{-1}$. inside the FOV is obtained by integrating along the line of sight. The dust emission is given by Eq. (3), but the number of grains of each size within the FOV is now a function of time. The dust local density is described as:

$N_{\text {dust }}(a)=\Delta Q_{\text {dust }}\left(t-\frac{r-r_{\mathrm{n}}}{v(a)}\right) \frac{n^{\prime}(a)}{4 \pi r^{2} v(a)}$

where $r$ is the radial distance from the center of the nucleus, $r_{\mathrm{n}}=3.0 \pm 0.1 \mathrm{~km}$ (Groussin et al. 2007) is the equivalent spherical radius of the nucleus, $n^{\prime}(a)$ is the grain size distribution of dust production, $\Delta Q_{\text {dust }}$ describes the time evolution of the dust production rate after impact, and $v(a)$ is the grain velocity. A Gaussian represents $\Delta Q_{\text {dust }}$ :

$\Delta Q_{\text {dust }}(t)=\exp \left(-\left(2 \sqrt{\ln (2)} \frac{t}{t_{\text {burst }}}\right)^{2}\right)$

where $t_{\text {burst }} / 2$ is the time scale at which the dust production is half of maximum, and $t$ is the time after impact. The velocity for each grain size is given by:

$v(a)=v_{\text {ref }}\left(\frac{a}{a_{\text {ref }}}\right)^{-q_{\mathrm{v}}}$

where $v_{\text {ref }}$ is the velocity for grain with radius $a_{\text {ref }}$ and $q_{\mathrm{v}}$ is the slope of the velocity distribution. For the power-law distribution $a_{\mathrm{ref}}$ is equal to $a_{\mathrm{min}}$, while for the Hanner distribution $a_{\mathrm{ref}}$ is equal to $a_{\mathrm{p}}$ (Harker et al. 2007). We determined $v_{\text {ref }}=230 \mathrm{~m} \mathrm{~s}^{-1}$ from the Spitzer SED observations assuming that the grains with $a_{\text {ref }}$ leave the FOV at TI $+3.6 \mathrm{~h}$ (Fig. 8). This value is consistent with the value of $220 \mathrm{~m} \mathrm{~s}^{-1}$ found by Harker et al. (2007) and other authors as reported by Ipatov \& A'Hearn (2011).

During the first few minutes after impact, the shadowing effect of the dust grains (caused by significant column density) was severe. Heating of grain material in the ejecta likely was delayed by $\approx 25$ min post-impact as self-shadowing diminished due to the expansion of the ejecta plume (DiSanti et al. 2007). Grains acquired most of their kinetic energy through momentum exchange with the gas released from subliming icy grains (Keller et al. 2007). The delay in dust acceleration was not taken into account in our modeling and produces $\simeq 10 \%$ error in our velocity estimate.

The temporal evolution of the ejecta dust emission at 5.7 and $12.5 \mu \mathrm{m}$ was computed for both amorphous carbon and twolayer grains, and compared to the fluxes at 5.7 and $12.5 \mu \mathrm{m}$ measured with Spitzer. The following results were obtained:

1. For the power-law size distribution, we used the $q$ and $a_{\mathrm{min}}$ values determined in Sect. 3.2 at TI $+0.67 \mathrm{~h}$. For the amorphous carbon grains, observations as the initial conditions at $t=0 \mathrm{~s}$ (impact), we fixed $a_{\min }=0.62 \mu \mathrm{m}$ and $q=3.3$. For the two-layer grains, we set $a_{\min }=0.1 \mu \mathrm{m}$ and $q=3.7$. The index $q_{\mathrm{v}}$ of the velocity distribution that best fit the decrease of the observed continuum flux at $\lambda=5.7 \mu \mathrm{m}$ with time is $0.55 \pm 0.05$ in the case of amorphous carbon, and $0.4 \pm 0.05$ for the two-layer composition (Fig. 8a). For the continuum at $\lambda=12.5 \mu \mathrm{m}$, the index $q_{\mathrm{v}}$ of the velocity distribution is $0.35 \pm 0.05$ in the case of amorphous carbon dust, and $0.4 \pm 0.05$ for grains with two-layer composition. The difference of the $q_{\mathrm{v}}$ may suggest that the velocity law is not a pure power-law over the size range sampled by the measurements.

2. The same approach was used when considering the Hanner distribution as measured at TI $+0.67 \mathrm{~h}$ (Sect. 3.2). For the amorphous carbon grains, at $t=0 \mathrm{~s}$ (impact), we fixed $a_{\mathrm{p}}=0.42 \mu \mathrm{m}$. For the two-layer grains, we set $a_{\mathrm{p}}=$ $0.1 \mu \mathrm{m}$. The index $q_{\mathrm{v}}$ of the velocity distribution that best-fits the decrease of the observed continuum flux at $\lambda=5.7 \mu \mathrm{m}$ with time is $0.4 \pm 0.05$ for both amorphous carbon and twolayer grains (Fig. 8b). For the continuum at $\lambda=12.5 \mu \mathrm{m}$, the index $q_{\mathrm{v}}$ of the velocity distribution is $0.3 \pm 0.05$ in the case of amorphous carbon grains, and $0.4 \pm 0.05$ for grains comprised of a two-layer composition.

The index $q_{\mathrm{v}}=0.40 \pm 0.05$ of the velocity distribution is consistent with the value of $q_{\mathrm{v}}=0.5$ expected for gas loading (Crifo \& Rodionov 1997).

The temporal decrease of the flux from $\mathrm{TI}+4.2 \mathrm{~h}$ to $\mathrm{TI}+10.2 \mathrm{~h}$ is well reproduced by our model (Fig. 8 ). However, the flux observed at TI $+20.5 \mathrm{~h}$ is underestimated by the model. The higher flux in data at $\mathrm{TI}+20.5 \mathrm{~h}$ could be explained by the production of small grains via fragmentation of large grains while they are still in the FOV. However, this possibility can not be substantiated with the Spitzer observations due to the lack of additional measurement of the coma SED between $\mathrm{TI}+10.2 \mathrm{~h}$ and $\mathrm{TI}+20.5 \mathrm{~h}$. Nucleus orientation and rotation state also are not adequately accounted for when subtracting the pre-impact spectrum from the TI $+20.5 \mathrm{~h}$ spectrum and a power-law velocity distribution may not be appropriate for the lowest velocities.

With the time-dependent model, we also created synthetic spectra of the ejecta using the size distributions determined at $\mathrm{TI}+0.67 \mathrm{~h}$ from the observations as the initial conditions at $t=0 \mathrm{~s}$. By fitting the synthetic spectra with the model described in Sect. 3.1, we determined the temporal evolution of the power law distribution and the Hanner distribution parameters $\left(a_{\min }, q\right.$, and $\left.a_{\mathrm{p}}\right)$. We find the parameters derived from fitting the observed spectra (Fig. 6) and from fitting the dust evolution model SEDs (Fig. 9) follow the same trends, though 

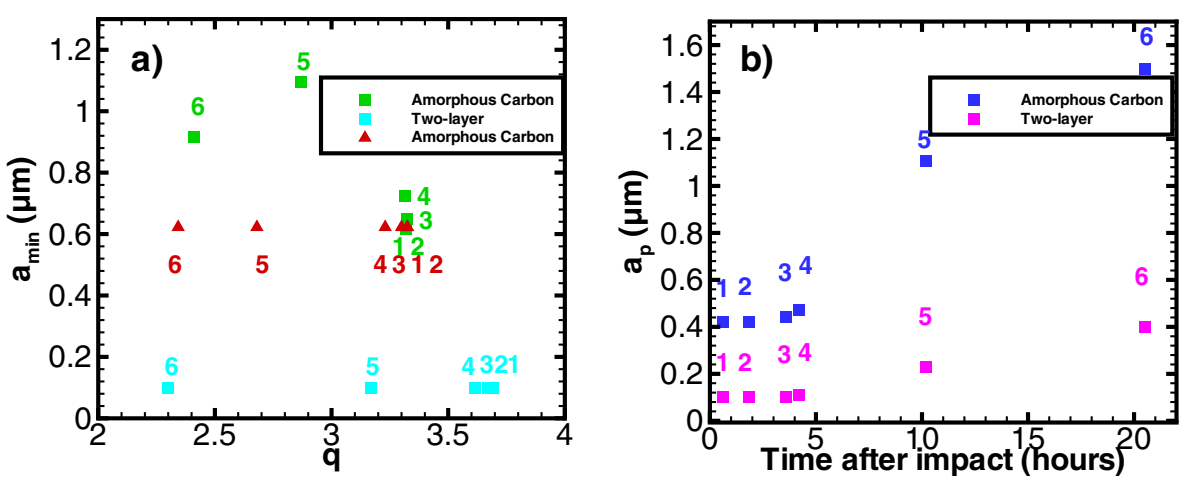

Fig. 9. Derived grain parameters for the power law distribution a) and for the Hanner distribution b) from the synthetic spectra of the time-dependent dust evolution model. The different times are labelled according to: (1) $\mathrm{TI}+0.67 \mathrm{~h}$; (2) $\mathrm{TI}+1.83 \mathrm{~h}$; (3) $\mathrm{TI}+3.6 \mathrm{~h}$; (4) $\mathrm{TI}+4.2 \mathrm{~h}$; (5) $\mathrm{TI}+10.2 \mathrm{~h}$; (6) $\mathrm{TI}+20.5 \mathrm{~h}$.

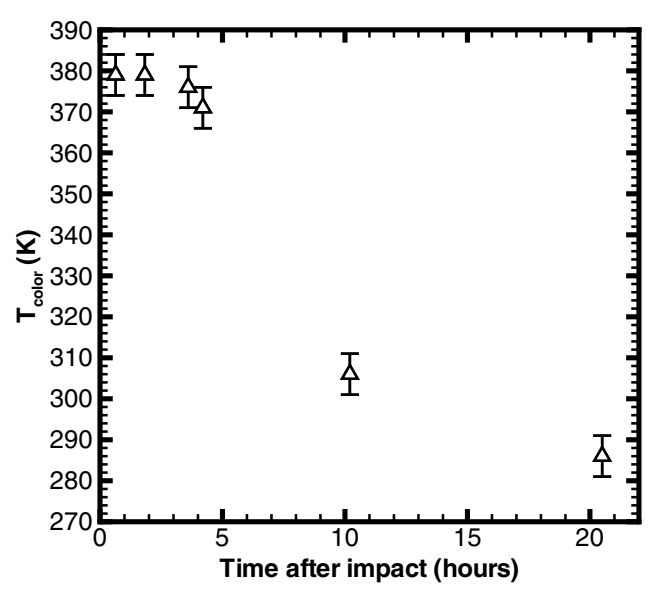

Fig. 10. Modeled temporal evolution of the color temperature $\left(T_{\text {color }}\right)$ of the ejecta within a $9.25^{\prime \prime} \times 9.25^{\prime \prime}$ FOV centered on the nucleus.

some differences are observed. Our dust evolution model predicts that $a_{\min }, q$ and $a_{\mathrm{p}}$ remain constant until TI $+3.6 \mathrm{~h}$ because all the grains in the ejecta population are still within the Spitzer FOV. For the Hanner distribution, as shown in Fig. 9b, $a_{\mathrm{p}}$ then increases with time, as observed (Fig. 6b), because the small grains leave the FOV faster than the large grains. In the case of the power law distribution (Fig. 9a) with $a_{\min }$ and $q$ as free parameters, the minimum grain size cut-off increases with time, contrary to the trend derived from modeling the observed SEDs (Fig. 6b). Interestingly, independent of the composition, the $q$ index is still decreasing (in both cases of $a_{\min }$ as a free parameter or not). At TI $+20.5 \mathrm{~h}$, for both compositions, $a_{\mathrm{p}}$ is higher in the time-dependent model than that deduced from the observations when the Hanner distribution is used, whereas $q$ is smaller in our model than that inferred from the actual observations if a power-law distribution is invoked to describe the grain population. Our interpretation of these outcomes is that the coma of comet 9P/Tempel 1 contains additional sources for small grains likely arising from fragmentation of large grains which are not accounted for in our time-dependent grain evolution model. This hypothesis is supported by the theoretical temporal evolution of $T_{\text {color }}$ (Fig. 10) obtained by fitting synthetic spectra as explained in Sect. 3 . At TI $+20.5 \mathrm{~h}$, the observed $T_{\text {color }}$ is $30 \mathrm{~K}$ higher than the computed value, suggesting an excess of hot small grains.

\section{Analysis of the $v_{2}$ water emission}

\subsection{Extraction of the number of water molecules}

The emission band detected in all spectra at $6.4 \mu \mathrm{m}$ primarily corresponds to fluorescence emission of the $v_{2}$ vibrational band of water (Woodward et al. 2007; Bockelée-Morvan et al. 2009). To measure the number of molecules of water within the FOV, the underlying continuum was first fit with a fourth order polynomial determined by the Levenberg-Marquardt method. Then the $v_{2}$ water band is highlighted by subtracting the polynomial fits to the spectra as shown in Fig. 11. We refer the reader to Woodward et al. (2007) for a detailed study of the synthetic spectral emission of the $v_{2}$ water band. The total intensity of the water band $\left(F_{v_{2}}\right)$ is then extracted over a range of wavelength that covers the width of the band.

Emission from the $v_{2}$ water band is, in first approximation, optically thin. Using the same approach as Feaga et al. (2007), we estimated that the optical thickness of the individual rovibrational lines is at most $\sim 0.3$ and 0.6 for pre-impact and post-impact data, respectively. In those calculations, we set the gas temperature to $30 \mathrm{~K}$ (DiSanti et al. 2007). The total band intensity is affected by less than $11 \%$ by optical depth effects ( $5 \%$ for the pre-impact data). For optically thin conditions, the number of molecules in the FOV is given by:

$N_{\mathrm{H}_{2} \mathrm{O}}=4 \pi \Delta^{2} \frac{F_{v_{2}}}{h v g_{v 2} r_{\mathrm{h}}^{-2}}$

where $h$ is the Planck constant and $g_{v 2}=2.41 \times 10^{-4} \mathrm{~s}^{-1}$ is the emission rate of $v_{2}$ at $r_{\mathrm{h}}=1 \mathrm{AU}$ (Bockelée-Morvan \& Crovisier 1989; Woodward et al. 2007).

We determined the number of water molecules within $9.25^{\prime \prime} \times 9.25^{\prime \prime}$ extraction apertures. The inferred $N_{\mathrm{H}_{2} \mathrm{O}}$ for the time before impact TI $-41.3 \mathrm{~h}$ is $(2.5 \pm 0.4) \times 10^{31}$ molecules for the extraction centered on the nucleus (Fig. 12). Just after impact $(\mathrm{TI}+0.67 \mathrm{~h}), N_{\mathrm{H}_{2} \mathrm{O}}$ increased by a factor 2.5 , remaining almost constant until TI $+3.6 \mathrm{~h}$. Figure 13 depicts the derived number of molecules in the impact, $N_{\mathrm{H}_{2} \mathrm{O}}$, as a funtion of time. The velocity of water molecules was measured to be about $0.75 \mathrm{~km} \mathrm{~s}^{-1}$ (Biver et al. 2007). Thus, these molecules should start to leave the FOV in less than 50 min and the amount of measured water should then decrease. The trend in Fig. 13 suggests that a nearly constant number of molecules $(\approx 3 \times$ $10^{31}$ molecules) persisted within the extraction aperture for upwards of $4 \mathrm{~h}$ prior to declining. This result strongly suggests that sustained production of water molecules occurred after impact from sublimating icy grains.

\subsection{Pre-impact water production rate}

For the pre-impact data, we performed $9.25^{\prime \prime} \times 9.25^{\prime \prime}$ extractions along the slit, at a number of offset positions with respect to the nucleus position. $N_{\mathrm{H}_{2} \mathrm{O}}$ values along the slit are shown in Fig. 12. In our model we assumed a spherical expansion of water with, an expansion velocity $v_{\exp }=0.75 \mathrm{~km} \mathrm{~s}^{-1}$ and a water 

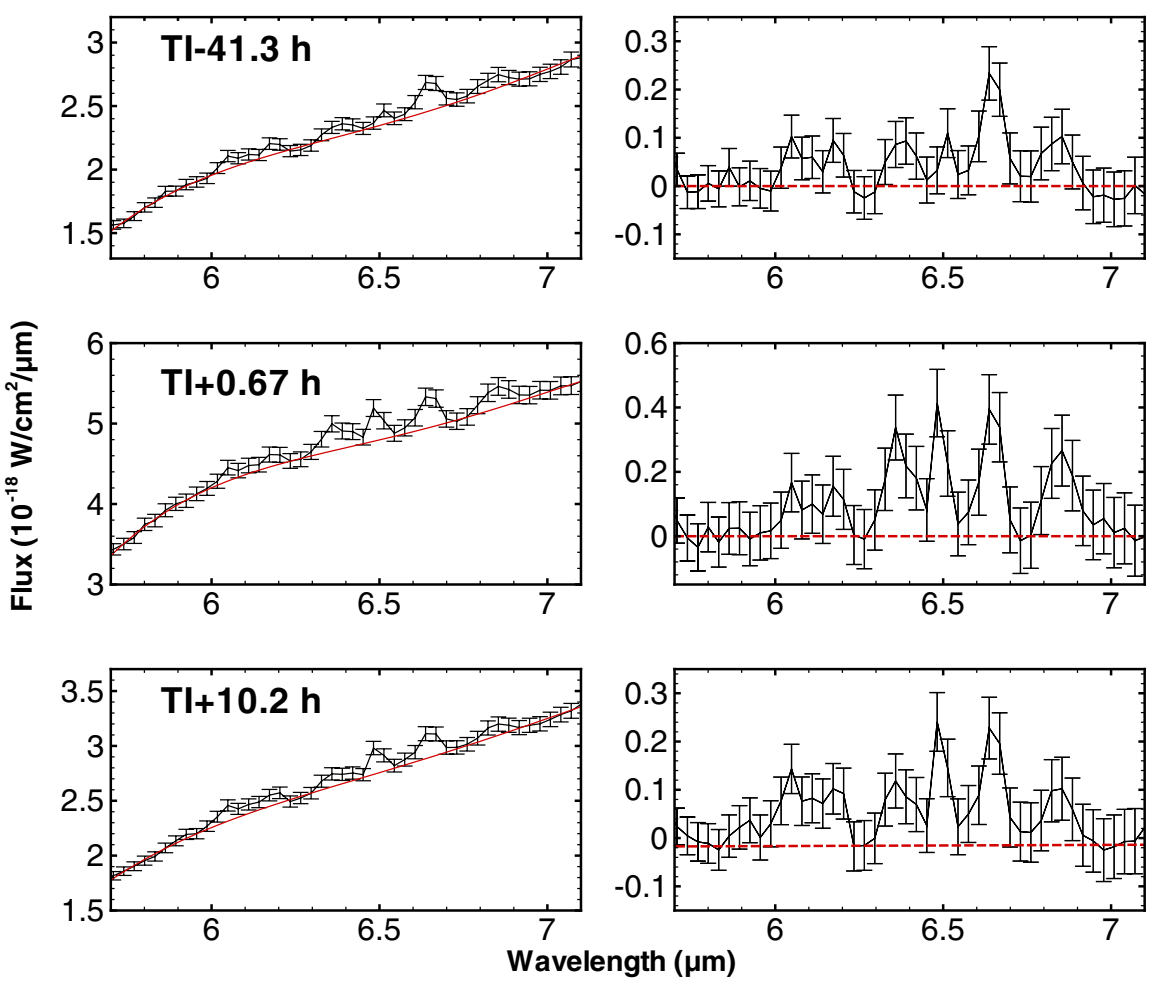

Fig. 11. Water spectra of comet 9P/Tempel 1 obtained by Spitzer. Left panel: polynomial fits to the underlying continuum are shown in red. Right panel: residual spectra showing water $v_{2}$ band emission.

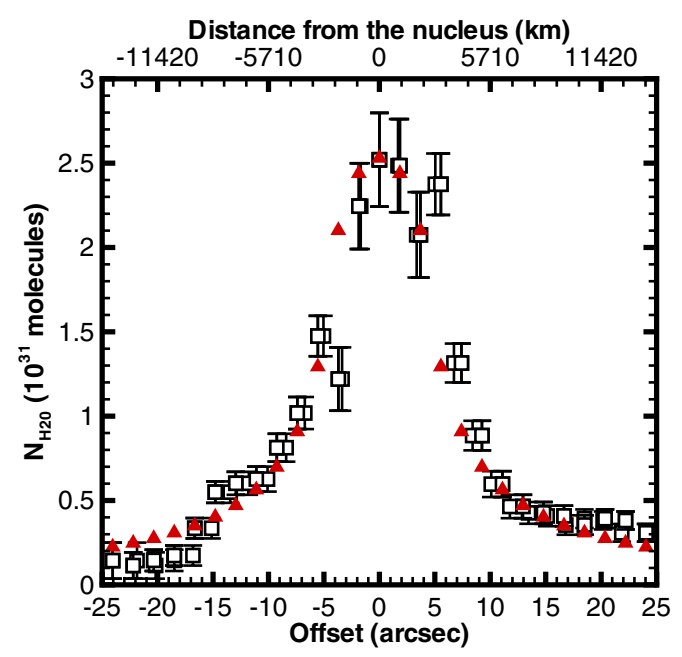

Fig. 12. Number of water molecules before impact (TI $-41.3 \mathrm{~h}$ ) along the length of the slit for the data (black) and our corresponding water model with $Q_{\text {pre }}=4.70 \times 10^{27} \mathrm{~mol} \mathrm{~s}^{-1}$ (red triangles). The FOV is $9.25^{\prime \prime} \times 9.25^{\prime \prime}$ for each extraction along the slit.

photodissociation rate $\beta=1.6 \times 10^{-5} \mathrm{~s}^{-1}$ at $r_{\mathrm{h}}=1 \mathrm{AU}$ (Crovisier 1989). Taking into account uncertainties in the measurement for the nucleus-centered extraction, the derived water production rate is $(4.7 \pm 0.7) \times 10^{27} \mathrm{~mol} \mathrm{~s}^{-1}$. Our model correctly reproduces the pre-impact spatial distribution of water molecules as shown in Fig. 12. Our water production rate is consistent with the water production rate determined by Küppers et al. (2005) and Feaga et al. (2007) but is a factor of two lower than that derived by DiSanti et al. (2007), Biver et al. (2007) and Feldman et al. (2007).

\subsection{Temporal evolution of water emission}

The temporal evolution of the number of water molecules within the FOV was investigated using a time-dependent water model.
This model assumes that the water molecules are produced from the very inner coma arising from an outburst. The physical process that produces the water molecules is not modeled here. In the next section, a physical model investigating sublimating ice grains will be presented. To generate the proper number of molecules to compare with the observed numbers, we integrate along the line of sight and the instrument FOV. The time-dependent water production rate is modeled with a Gaussian function similar to the description used in the timedependent dust model (Sect. 3.4). The number of molecules is evaluated from:

$N_{\mathrm{H}_{2} \mathrm{O}}(t)=\int_{V} \frac{\Delta Q_{\mathrm{H}_{2} \mathrm{O}}(r, t) \mathrm{e}^{-r / L_{\mathrm{H}_{2} \mathrm{O}}}}{4 \pi r^{2} v_{\exp }} \mathrm{d} V$

where $V$ is the volume of integration along the FOV, and $\Delta Q_{\mathrm{H}_{2} \mathrm{O}}$ describes the Gaussian outburst

$\Delta Q_{\mathrm{H}_{2} \mathrm{O}}(r, t)=Q_{\text {post }} \exp \left(-\left(2 \sqrt{\ln (2)} \frac{t-\left(r-r_{\mathrm{n}}\right) / v_{\text {exp }}}{t_{\text {burst }}}\right)^{2}\right)$

where $Q_{\text {post }}$ is the water production rate just after impact and $L_{\mathrm{H}_{2} \mathrm{O}}$ is the photodissociation scale length.

Figure 13 shows the result of this time-dependent model. With a short-duration outburst $\left(t_{\text {burst }}=500 \mathrm{~s}\right)$, the strong production of water that is observed just after impact (TI $+0.67 \mathrm{~h}$ ) is well reproduced by our model. To explain the sustained production of water after TI $+0.67 \mathrm{~h}$, a long-duration outburst is required. We estimate the time scale of this outburst equal to $t_{\text {burst }}=70000 \mathrm{~s}(19.4 \mathrm{~h})$. DiSanti et al. (2007) observed that the $\mathrm{H}_{2} \mathrm{O}$ column density started to increase abruptly $25 \mathrm{~min}$ after impact. Consideration of this delay within our model does not substantially affect our model results. A long term outburst can be explained by the sublimation of long-lived icy grains near the nucleus outflowing at low velocities. The best fit to the observational data is achieved by modeling the outburst as a linear combination of the short-duration outburst and the long-duration 


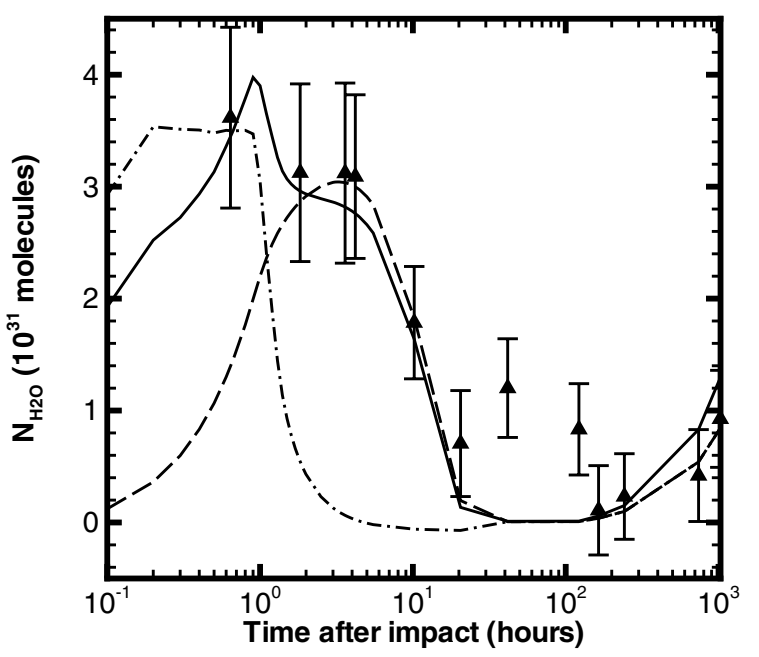

Fig. 13. Number of water molecules in the ejecta measured from the data (triangle) for a $9.25^{\prime \prime} \times 9.25^{\prime \prime}$ (corresponding to an area $\sim 4900 \times$ $4900 \mathrm{~km}$ within the coma) aperture centered on the nucleus. Number of water molecules expected for a short-duration outburst $t_{\text {burst }}=$ $500 \mathrm{~s}$ (dash-dotted line), a long-duration outburst $t_{\text {burst }}=70000 \mathrm{~s}$ (long-dashed line) and a linear combination of the short-duration outburst and the long-duration outburst (solid line) with a relative proportion of water molecules injected during the short-duration outburst of 0.13 .

outburst with a relative proportion of water molecules injected during the short-duration outburst of 0.13 (Fig. 13).

The number of water molecules injected by the impact from our model is $\simeq(2.5 \pm 0.5) \times 10^{32}$ molecules which corresponds to a mass of water of $(7.4 \pm 1.5) \times 10^{6} \mathrm{~kg}$. This result is in good agreement with the mass of $(6.8 \pm 2.3) \times 10^{6} \mathrm{~kg}$ as reported by Keller et al. (2007). Interestingly, the energy delivered by the Deep Impact impactor $\left(1.9 \times 10^{10} \mathrm{~J}\right.$; A'Hearn et al. 2005) is insufficient (by a factor 840) to vaporize enough ice to produce this mass of water. DiSanti et al. (2007) and Groussin et al. (2010) argue that the principal source of volatiles in the ejecta was sublimation from outflowing grains. The time delay in the production of volatiles observed by DiSanti et al. (2007) is presumably due to delayed grain heating caused by the initial optical thickness of the dust cloud.

\subsection{Water from icy grains}

\subsubsection{Model of the sublimation of icy grains}

For a quantitative analysis of the sublimation of icy outflowing grains we considered four different grain compositions: pure water ice grains, two-layer $(\mathrm{Ol})$ grains consisting of a silicate core covered by water ice, two-layer (C) grains consisting of a carbon core covered by water ice, and three-layer grains consisting of a silicate core surrounded by an organic component, covered by a mantle of water ice. The fractional mass of the organic component encasing the silicate core, $\alpha$, was set to 0.1 . The fractional mass of the water component, $\alpha_{\text {ice }}$, is 0.98 for the two-layer grains, and 0.90 for the three-layer grains. Hence, we investigated icy grains with various ice contents and thermal properties. We used the real and imaginary indices of hexagonal ice given by Warren (1984).

In our dust thermal model described in Sect. 3.1, the temperature of a grain was derived by assuming the equilibrium between the energy absorbed from the Sun and the energy reradiated in the infrared. However, for icy grains, the cooling by sublimation $\left(E_{\text {subl }}\right)$ cannot be neglected. $E_{\text {subl }}$ can be expressed as follows:

$E_{\text {subl }}=H\left(T_{\mathrm{d}}\right) Q_{\mathrm{H}_{2} \mathrm{O}}\left(T_{\mathrm{d}}\right)$

where $H$ is the latent heat of sublimation of water ice $\left(\mathrm{J} \mathrm{kg}^{-1}\right)$ related to the temperature of the grains through (Delsemme \& Miller 1971):

$H\left(T_{\mathrm{d}}\right)=2.88810^{6}-1116 T_{\mathrm{d}}$.

The water production rate, $Q_{\mathrm{H}_{2} \mathrm{O}}\left(\mathrm{kg} \mathrm{s}^{-1}\right)$ is given by:

$Q_{\mathrm{H}_{2} \mathrm{O}}\left(T_{\mathrm{d}}\right)=4 \pi a^{2} P_{\mathrm{v}}\left(T_{\mathrm{d}}\right) \sqrt{\frac{m_{\mathrm{H}_{2} \mathrm{O}}}{2 \pi k_{\mathrm{B}} T_{\mathrm{d}}}}$

where $m_{\mathrm{H}_{2} \mathrm{O}}$ is the mass of the water molecule, $k_{\mathrm{B}}$ is the Boltzmann constant and $P_{\mathrm{v}}$ is the vapor pressure derived from Lichtenegger \& Komle (1991):

$P_{\mathrm{v}}\left(T_{\mathrm{d}}\right)=P_{r} \exp \left[\frac{m_{\mathrm{H}_{2} \mathrm{O}} L}{k_{\mathrm{B}}}\left(\frac{1}{T_{r}}-\frac{1}{T_{\mathrm{d}}}\right)\right]$

where $P_{r}=10^{5} \mathrm{~N} \mathrm{~m}^{-2}, T_{r}=373 \mathrm{~K}$ and $L=2.78 \times 10^{6} \mathrm{~J} \mathrm{~kg}^{-1}$.

The evolution of the grains, once they are ejected into the coma, depends on their initial size, their composition and the heliocentric distance. We follow the evolution of grains until the icy layer sublimates completely. The variation of the grain radius with time due to the sublimation is given by:

$\left|\frac{\mathrm{d} a}{\mathrm{~d} t}\right|=\frac{P_{\mathrm{v}}\left(T_{\mathrm{d}}\right)}{\rho_{\mathrm{d}}} \sqrt{\frac{m_{\mathrm{H}_{2} \mathrm{O}}}{2 \pi k_{\mathrm{B}} T_{\mathrm{d}}}}$.

The sublimation results in mass loss expressed as:

$\frac{\mathrm{d} m_{\mathrm{ice}}}{\mathrm{d} t}=-Q_{\mathrm{H}_{2} \mathrm{O}}$

The grain lifetime, $t_{\text {grain }}$, is the time needed for an icy layer of grains to sublimate completely. Depending on the chemical composition of grains, i.e., pure ice or mixtures, their thermal properties vary as carbon and olivine absorb much more radiation than water ice. Thus, the sublimation is more efficient in the case of two or three-layer grains than for the pure water ice. The lifetime of the dirty icy grains is then shorter than the one for the water ice case such that the lifetimes of the grains in increasing order according to composition are: two-layer $(\mathrm{C})$, threelayer, two-layer $(\mathrm{Ol})$, and pure-ice. The lifetime of grains is presented in Fig. 14. The lifetimes of the two-layer (C) grains with $\alpha_{\text {ice }}=0.80$ and 0.90 are similar within $10 \%$. Hence, the lifetime of dirty grains is not much sensitive to the exact amount of "dirt" contamination with the ice as asserted by Beer et al. (2006). Beer et al. (2006) obtained similar grain temperatures for mixtures of ice and tholins with $\alpha_{\text {ice }}=0.5$ and $\alpha_{\text {ice }}=0.1$.

\subsubsection{Dynamical expansion}

To compute the temporal evolution of water production, we assume that the grains, of radii $0.1-100 \mu \mathrm{m}$, are produced instantaneously, i.e., as for a short-duration outburst, and are moving radially with a velocity dependent of the initial grain radius given by Eq. (13). For the grain velocity we used $v_{\text {ref }}=230 \mathrm{~m} \mathrm{~s}^{-1}$ and $q_{\mathrm{v}}=0.4$, adopting a power law distribution for the grains with $a_{\min }=0.1 \mu \mathrm{m}$ and $q=3.7$.

We used two approaches to account for the dynamics of water molecules escaping from grains. In the first approach, 


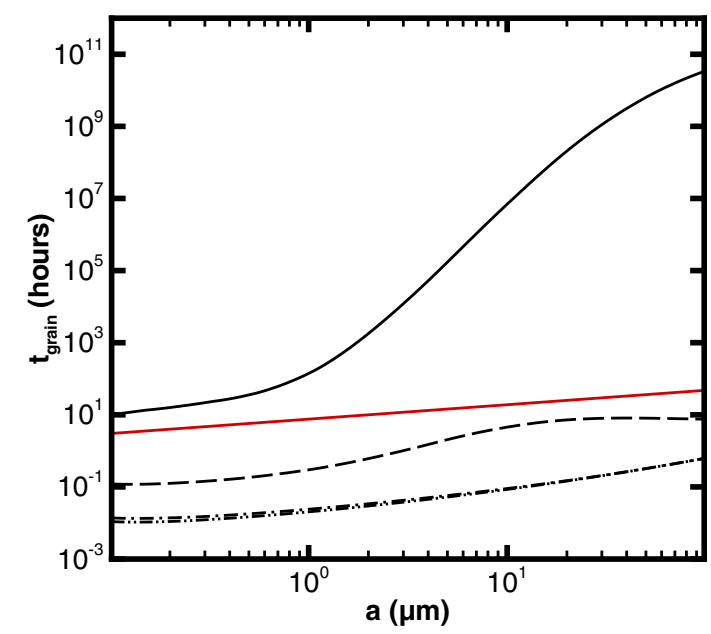

Fig. 14. Grain lifetimes regarding sublimation: pure ice grains (solid line); three-layer grains with a fractional mass of the mantle component $\alpha_{\text {ice }}=0.90$ (dash-dotted-dotted line); two-layer $(\mathrm{Ol})$ grains with a core of amorphous silicate and a mantle of water ice with $\alpha_{\text {ice }}=0.98$ (long-dashed line); two-layer (C) grains with a core of amorphous carbon and a mantle of water ice with $\alpha_{\text {ice }}=0.98$ (dash-dotted line). The dash-dotted-dotted line and the dash-dotted line are nearly identical. The grain residence time within the $9.25^{\prime \prime} \times 9.25^{\prime \prime}$ FOV is shown by the red line. We assumed $v_{\text {ref }}=230 \mathrm{~m} \mathrm{~s}^{-1}$ and $q_{\mathrm{v}}=0.4$.

the water molecules ejected from the grains, are moving radially with respect to the nucleus frame with a fixed velocity equal to the gas velocity. In the second approach, water molecules are sublimating isotropically from the grains. The velocity of the water molecule is selected randomly from a half-Maxwell distribution function at the grain temperature, $T_{\mathrm{d}}$. Once produced, the molecules are not subject to collisions. The space surrounding a given grain is subdivided into cells with small volumes. Molecules appearing within a cell are counted over a time duration commensurate with the Spitzer observational intervals to generate model statistics. We can then derive the spatial distribution of water number density and the mean $\mathrm{H}_{2} \mathrm{O}$ velocity. Both models provide, for the various measurement times, the spatial distribution of water molecule populations (both the isotropic and radially sublimated cases) produced by the different grain sizes. These two models are complementary: the first one is appropriate for a dense medium where the water molecules sublimating from grains are forced, by collisions with the ambient flow, to move in the same radial direction. In the second case (analogous to the vectorial model for photodissociation products), the medium is rarefied, collisions are less important, and the water molecules are freely expanding isotropically from grains. Our time-dependent, $\mathrm{H}_{2} \mathrm{O}$ production numbers within the extraction aperture FOV are presented in Fig. 15.

\subsubsection{Results}

The observed water production requires a combination of pure water ice grains and dirty grains (Fig. 15). Pure water ice grains cannot fully explain the observed water production (Fig. 15). However, the overall temporal evolution at $\mathrm{TI}+1.83 \mathrm{~h}$ and later is well reproduced by the sublimation of pure water ice grains. Models with three-layer grains can account for the number of water molecules detected at TI $+0.67 \mathrm{~h}$; on the other hand, these models predict a fast decrease of water production. The contribution of the grains to the water production also depends on their size. In the case of pure ice, only grains with

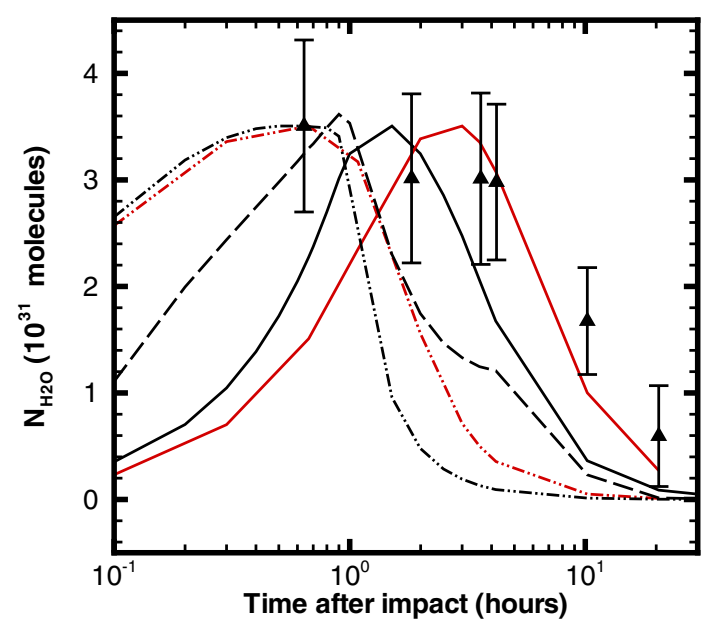

Fig. 15. Temporal evolution of the number of water molecules within the FOV computed with our time-dependent models of sublimating icy grains. Curves are displayed for pure ice grains (solid line), three-layer grains with a fractional mass of the mantle component $\alpha_{\text {ice }}=0.9$ (dashdotted-dotted line) and two-layer (Ol) grains with $\alpha_{\text {ice }}=0.98$ (longdashed line). The outputs from the models with rarefied medium and dense medium are shown in red and black, respectively. The filled triangles are the number of water molecules derived from the analysis of the Spitzer spectra. The model results have been adjusted so that the curves peak at $(3.5 \pm 0.8) \times 10^{31}$ molecules, which corresponds to the observed value at $\mathrm{TI}+0.67 \mathrm{~h}$.

radii between $0.1-0.6 \mu \mathrm{m}$ lose their ice mantle before they leave the FOV at TI $+3.6 \mathrm{~h}$. In contrast, the lifetime of dirty grains does not exceed $1 \mathrm{~h}$ for $a<100 \mu \mathrm{m}$; grains with radii between $0.1-100 \mu \mathrm{m}$ and even larger are totally vaporized before they leave the FOV and so the temporal evolution of $\mathrm{N}_{\mathrm{H}_{2} \mathrm{O}}$ from these grains is essentially set by the gas velocity. The two-layer $(\mathrm{Ol})$ grain model (with a core of amorphous silicates and a mantle of water ice with $\alpha_{\text {ice }}=0.98$ ) is an intermediate case between the three-layer and the pure water ice extremes. In Fig. 15 we provide, for comparison an example of the simulated temporal evolution of the water evolution for the two-layer $(\mathrm{Ol})$ case. As expected, for these grains the time evolution of the number of molecules is between the three-layer and the pure water ice grain scenarios.

Slowly moving, fine grained $(1 \pm 1 \mu \mathrm{m})$ water ice particles, free from refractory impurities, were detected from the Deep Impact spacecraft (Sunshine et al. 2007). The presence of pure ice grains in the ejecta is also in agreement with the change of the IR color of the dust coma after impact (Knight et al. 2007; Fernández et al. 2007).

In our model, we assumed that the dust grains move radially from the nucleus in a radially expanding ambient gas flow. We investigated two cases of gas flow: dense and rarefied. In the dense case, water molecules after sublimation from grains are forced by collisions with the ambient flow to move in the same radial direction and with the same velocity. Therefore, in this case, we have a maximum outflow rate. In the rarefied medium scenario, collisions are rare, and molecules emitted from grains travel isotropically. Thus the average radial flow rate is smaller than in the previous case, and $N_{\mathrm{H}_{2} \mathrm{O}}$ decreases at later times. Until $\mathrm{TI}+0.67 \mathrm{~h}$, the two approaches give the same $N_{\mathrm{H}_{2} \mathrm{O}}$ because no water molecules have left the FOV. In the case of pure water ice, the maximum $N_{\mathrm{H}_{2} \mathrm{O}}$ is reached much later, when water molecules have already started to leave the FOV. In the dense medium case, we deduce a mass of ice $M_{\text {ice }}=7.6 \times 10^{6} \mathrm{~kg}$ (for grains between $0.1-1 \mu \mathrm{m}$ ) if only pure water ice grains are 
A. Gicquel et al.: Dust and ice in comet 9P/Tempel 1 from Spitzer observations

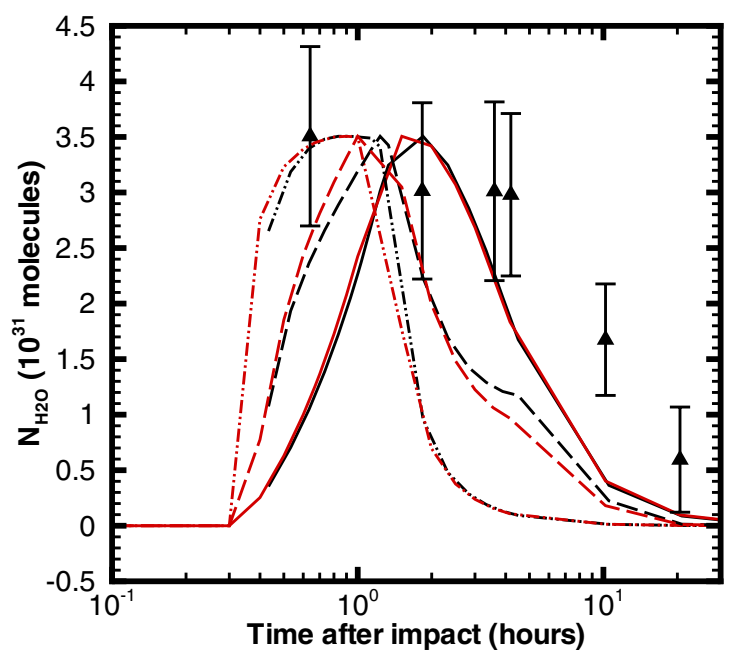

Fig. 16. Same as Fig. 15 for the dense medium case, taking into account a delay of $20 \mathrm{~min}$ in the sublimation only (red curves), and in the sublimation and acceleration of the grains (black curves).

considered (Table 6) and $M_{\text {ice }}=3.6 \times 10^{5} \mathrm{~kg}$ (for grains between $0.1-100 \mu \mathrm{m}$ ) when only the three-layer grains are used (Table 5). In the rarified medium model, we find $M_{\text {ice }}=4.7 \times 10^{6} \mathrm{~kg}$ (for grains between $0.1-1 \mu \mathrm{m}$ ) in the case of pure water ice grains and $M_{\text {ice }}=3.5 \times 10^{5} \mathrm{~kg}$ (for grains between $0.1-100 \mu \mathrm{m}$ ) in the case of the three-layer grains (Table 5).

We have assumed that the sublimation commenced immediately at the time of impact. However, DiSanti et al. (2007) find that the column abundance of $\mathrm{H}_{2} \mathrm{O}$ increased by only a modest factor until TI $+25 \mathrm{~min}$. They assert that this time delay is required for outflowing grains to reach sublimation temperature in an optically thick dust coma. We studied the effect of this delay in the dense case and considered two limiting cases. First (1st case), we assumed that the grains after impact achieved velocities close to those arising from aerodynamic acceleration. For this case, we delayed the onset of grain sublimation by $20 \mathrm{~min}$, while the grains are still moving outwards into the coma. Then sublimation commenced from the grain position (i.e., total displacement from the nucleus at $t=20 \mathrm{~min}$ ). Alternatively ( 2 nd case), we considered a case wherein the grains after the DI ejection event acquired a low velocity (i.e., they were not entrained in the initial very high velocity plume ejected from the impact site) and therefore stayed relatively close to the nucleus for the first $20 \mathrm{~min}$. For simplicity, to simulate this scenario in the dense case, we took the results of the prior simulations (Fig. 15), shifted by $20 \mathrm{~min}$. We find that the variation of $N_{\mathrm{H}_{2} \mathrm{O}}$ in the $\mathrm{FOV}$ with time is quite similar for the two cases (Fig. 16). The reason for this consonance is that in $20 \mathrm{~min}$, the largest grains and the smallest grains pass respectively the distances of $20 \mathrm{~km}$ and $280 \mathrm{~km}$ from the nucleus (1\% and $10 \%$ of the FOV respectively). Therefore, the water molecules start to leave the FOV 15 min later than the model without the delay and the time when $N_{\mathrm{H}_{2} \mathrm{O}}$ starts to decrease is shifted.

The plateau observed between $\mathrm{TI}+0.67 \mathrm{~h}$ and $\mathrm{TI}+4.2 \mathrm{~h}$ in our data reveals a continuous $\mathrm{H}_{2} \mathrm{O}$ production that is not reproduced with either the pure-ice or three-layer simulations. We found that a linear combination of these simulations is not able to fit the data. A more complex model that considers a large variety of grain properties and ice content is beyond the scope of this paper.

Finally, we have investigated whether we could reproduce, with our model, the temporal evolution of the water column
Table 4. Set of parameters used in the modeling.

\begin{tabular}{lcccc}
\hline \hline $\begin{array}{l}\text { Parameter } \\
\text { set }\end{array}$ & $\begin{array}{c}v_{\text {ref }} \\
\left(\mathrm{m} \mathrm{s}^{-1}\right)\end{array}$ & $q_{\mathrm{v}}$ & $\begin{array}{c}a_{\min } \\
(\mu \mathrm{m})\end{array}$ & $\begin{array}{c}\text { Size distribution } \\
\text { parameter }\end{array}$ \\
\hline$(1)$ & 230 & 0.4 & 0.1 & $q=3.7$ \\
$(2)$ & 230 & 0.55 & 0.62 & $q=3.3$ \\
$(3)$ & 230 & 0.4 & 0.1 & $a_{\mathrm{p}}=0.1 \mu \mathrm{m}$ \\
$(4)$ & 230 & 0.4 & 0.1 & $a_{\mathrm{p}}=0.42 \mu \mathrm{m}$ \\
\hline
\end{tabular}

Table 5. Required mass of ice injected in the dense medium case and in the rarefied medium for grains with radii between $0.1-100 \mu \mathrm{m}$.

\begin{tabular}{lccc}
\hline \hline $\begin{array}{l}\text { Parameter } \\
\text { set }\end{array}$ & $\begin{array}{c}\text { Grain } \\
\text { composition }\end{array}$ & dense & $M_{\text {ice }}$ \\
\hline$(1)$ & pure water ice & $2.30 \times 10^{7}$ & $1.49 \times 10^{7}$ \\
& two-layer (Ol) & $1.18 \times 10^{6}$ & - \\
& three-layer & $3.58 \times 10^{5}$ & $3.48 \times 10^{5}$ \\
\hline$(2)$ & pure water ice & $4.65 \times 10^{10}$ & - \\
& two-layer (C) & $1.48 \times 10^{7}$ & - \\
\hline$(3)$ & pure water ice & $2.30 \times 10^{7}$ & - \\
& two-layer (Ol) & $1.18 \times 10^{6}$ & - \\
& three-layer & $3.58 \times 10^{5}$ & - \\
\hline$(4)$ & pure water ice & $5.37 \times 10^{8}$ & - \\
& two-layer (C) & $2.73 \times 10^{6}$ & - \\
\hline
\end{tabular}

Notes. The model results have been adjusted so that the curves peak at $(3.5 \pm 0.8) \times 10^{31}$ molecules, which corresponds to the observed value at $\mathrm{TI}+0.67 \mathrm{~h}$.

abundance measured at Keck by DiSanti et al. (2007). The data acquired by DiSanti et al. (2007) pertain to a much (typically ten times) smaller field of view $(280 \mathrm{~km} \times 1130 \mathrm{~km})$ and samples $\sim 12$ times until TI $+1.5 \mathrm{~h}$. Using the number of pure ice grains required to reproduce the long-term evolution of the $\mathrm{H}_{2} \mathrm{O}$ molecules observed by Spitzer, we infer a maximum column abundance for the Keck data that is three times smaller than the maximum observed column abundance. If one uses instead the number of three-layer grains required to explain the Spitzer data at TI $+0.67 \mathrm{~h}$, the Keck column abundance is overestimated by a factor of about 2 ; in addition the overall temporal evolution is not reproduced. On the other hand, the twolayer $(\mathrm{Ol})$ grains assumption approximately fits the $\mathrm{H}_{2} \mathrm{O}$ column abundance mesured at Keck and its temporal evolution.

So far we presented the results obtained using the parameters for the power law distribution $\left(a_{\min }=0.1 \mu \mathrm{m}\right.$ and $\left.q=3.7\right)$ and for the grain velocity $\left(v_{\text {ref }}=230 \mathrm{~m} \mathrm{~s}^{-1}\right.$ and $\left.q_{\mathrm{v}}=0.4\right)$ obtained by fitting the dust continuum at $\mathrm{TI}+0.67 \mathrm{~h}$ with the thermal emission of two layers grains. A similar temporal evolution of $N_{\mathrm{H}_{2} \mathrm{O}}$ is obtained for the three other size distributions discussed in Sect. 3 and summarized in Table 4. The mass of ice required to explain the $N_{\mathrm{H}_{2} \mathrm{O}}$ measured at TI $+0.67 \mathrm{~h}$ (case of dirty grains) or the sustained $N_{\mathrm{H}_{2} \mathrm{O}}$ value measured at later times (taken to be $3.5 \times$ $10^{31}$ molecules; case of pure ice grains) is given in Table 5 for the different parameter sets with $a_{\max }=100 \mu \mathrm{m}$. Sets (1) and (3) provide identical masses of ice because they correspond to the same size and velocity distribution parameters (see Sect. 3.2 and Table 4). A huge unrealistic mass of ice is inferred with set (2) where $a_{\min }=0.6 \mu \mathrm{m}$ (Table 5). The sublimation of pure ice grains larger than $0.6 \mu \mathrm{m}$ is indeed inefficient.

Since sublimation from pure ice grains larger than $0.6 \mu \mathrm{m}$ is inefficient, and the lifetime of $0.1 \mathrm{um}$ pure ice grains are short, the major source of water molecules in the Spitzer beam probably are pure ice grains with radii between $0.1-0.6 \mu \mathrm{m}$. 
Dirty icy grains over a large size range contribute to some extent at early times. The mass of ice in those grains can be neglected in the total ice budget.

Our model and analysis of dust thermal emission did not consider icy grains. We computed the thermal emission expected from pure ice grains and found that its contribution to the continuum spectral energy distribution observed by Spitzer is negligible. The ratio between the thermal flux from carbon grains and from pure ice grains is close to 100 . The dirty ice grains sublimated quickly (Fig. 14) and do not affect models that seek to replicate the observed Spitzer spectra. Consequently, there was indeed no need to include water ice in our analysis of the dust continuum emission.

\subsection{Dust-to-ice ratio}

We summarize in Table 6 the masses of dust and ice in the ejecta cloud inferred for the different set of grain parameters and for the size ranges $0.1-1,0.1-10$, and $0.1-100 \mu \mathrm{m}$. We only consider pure ice grains in the ice budget.

The dust-to-ice ratio (Table 6) was computed assuming that the size distributions of ice and dust grains are similar. The dustto-ice ratio is $<0.02$ in all cases. For comparison, the dust-to-gas production rate ratio before impact is $\sim 2$ based on the pre-impact water production rate obtained in Sect. $4.2\left(141 \mathrm{~kg} \mathrm{~s}^{-1}\right)$, and the pre-impact dust production rate $293 \mathrm{~kg} \mathrm{~s}^{-1}$ (Schleicher et al. 2006 ). With a ice-to-dust ratio of $\approx 50$ for sizes $<100 \mu \mathrm{m}$, we could conclude that a large amount of ice was located below the impacted surface of 9P/Tempel 1. However this ice-to-dust ratio seems non-realistic because, e.g., this would imply $\mathrm{O} / \mathrm{Mg}$ and $\mathrm{O} / \mathrm{Si}$ bulk elemental abundances inside the nucleus much higher than protosolar values. Because the dust mass in this study is in good agreement with other published values (Sect. 3.3), we investigated how to decrease the mass of ice.

In our study, we considered dirty ice grains with radii less than $100 \mu \mathrm{m}$. We verified that considering larger grains do not affect our conclusions. Although these slowly moving grains vaporize close to the nucleus, they cannot explain the amount of water observed well after impact. We can explain the sustained water production by significantly reducing the gas velocity, but this explanation is not realistic given observational constraints on the gas velocity field (Biver et al. 2007). In our calculations, we assumed that sublimation is occurring from the spherical surface of the grains. In more realistic models (Gunnarsson 2003), porous grains are assumed to be aggregates of small particles and so the effective surface area of sublimation is larger than the surface area defined by the grain radius. To test how this would affect our results, we performed calculations for porous grains made of units of $0.2 \mu \mathrm{m}$ grains. For aggregate radii of $0.5 \mu \mathrm{m}$ and $100 \mu \mathrm{m}$ radii, the effective surface area of sublimation is 1.5 and 75 times larger than the surface area defined by the grain radius. Use of porous grains results in ice masses that are only $\sim 10 \%$ lower than dirty ice grains. Finally, in our model we assumed a spherical expansion of the ejecta cloud for simplification. To investigate geometry effects, we used the model for the rarefied medium and assumed that the ejecta expanded in a cone of 45 degrees opening angle along the line of sight. We obtained a mass of ice only $15 \%$ smaller than the value inferred in the isotropic geometry. In summary, it is difficult to decrease the estimated mass of ice by more than a factor 2 with the above arguments.

Possibly, our assumption that the size distribution for the dust and icy grains is similar is not appropriate. Infrared ice signatures in ejecta spectra suggest that the size of pure ice grains is in the range $1 \pm 1 \mu \mathrm{m}$ (Sunshine et al. 2007). By assuming a smaller grain size cut-off for icy grains than for dust grains (which could be due to the rapid fragmentation of ice grains after impact), we can deduce a more realistic dust-to-ice ratio. For example, using the total dust mass inferred for grains $<100 \mu \mathrm{m}$ and $<10 \mathrm{~mm}$ and the mass of ice for grains $<1 \mu \mathrm{m}$, we obtain dust-toice ratios of $\approx 0.1$ and 0.3 , respectively. The comparison with the pre-impact dust-to-gas production rate ratio of $\sim 2$ would then indicate an ice enrichment by a factor $>6$ below the surface.

A third possible interpretation involves ice grains that are on gravitationally bound trajectories. Our model has assumed that all observed water and dust is escaping from the gravitational influence of the nucleus. This assumption is incorrect for a gravity-dominated cratering event, which is not ruled out by observational data (Richardson et al. 2007). Richardson et al. (2007) point out that, in this case, more than $90 \%$ of the mass excavated by the impactor never travels more than a few hundred meters from the surface. This material returns to the surface within 45 min after impact, which is approximately the time of our first Spitzer spectral map. Fifty percent of this material is ejected very slowly and lands within 9 min over a $160 \mathrm{~m}$ diameter area. In fact, slow moving material continues to fall back onto the surface for many hours. It is important to recognize that this redistributed material will contain icy grains. Upon reaching the surface these icy grains can be warmed by direct insolation or through thermal contact with surrounding dust. Therefore, an alternate production mechanism of water vapor arises from pure and dirty ice grains that have been redistributed over the surface of the comet and sublime. If one assumes that those grains exhausted their whole water content, i.e., $7.4 \times 10^{6} \mathrm{~kg}$ of water (Sect. 4.3 ), and that $10 \%$ of the excavated material escaped in the coma, then we obtain a dust-to-ice ratio for the excavated material in the range $0.6-2.5$ (i.e., an ice-to-dust ratio of 0.4-1.6), consistent with the preimpact dust-to-gas production rate ratio of $\sim 2$. In this calculation, we use the dust mass we determined when assuming a maximum dust size in the ejecta cloud of $100 \mu \mathrm{m}$. Larger dustto-ice values are derived if larger maximum sizes are allowed, as considered by Küppers et al. (2005). However, by modeling the ejecta plume behavior, Richardson et al. (2007) estimated the excavated mass to be at most $1.8 \times 10^{7} \mathrm{~kg}$ (the maximum value being obtained for a gravity-dominated cratering event), implying a dust-to-ice ratio $<1.4$. In summary, in the hypothesis of a gravity-dominated cratering event and complete sublimation of excavated ice, the dust-to-ice ratio is estimated to $0.4-1.4$, implying at most a moderate enrichment in water ice below the surface with respect to the pre-impact $\mathrm{H}_{2} \mathrm{O}$ /dust production rate ratio. The question that then arises is whether the observed time evolution of $N_{\mathrm{H}_{2} \mathrm{O}}$ is consistent with sublimation from redeposited material. Assuming that $50 \%$ of the observed water was produced from the material that landed over a region of $160 \mathrm{~m}$ in diameter, the sustained water production observed by Spitzer (Fig. 13) implies that this material remained in average at an equilibrium temperature on the order of 230-240 K, i.e., significantly below the surface temperature of the impacted area $(\sim 300 \mathrm{~K}$, Groussin et al. 2007). If these suppositions are to be upheld, pure ice grains would have to be present in the ejecta blanket and would have to had remained colder than the surrounding nuclear surface. Extensive modeling of the sublimation of the ejecta blanket is beyond the scope of this paper. In addition, whether the cratering event was gravity or strength-dominated is still debated (Holsapple \& Housen 2007), so that the amount of material that returned back to the surface is not precisely known. 
Table 6. Total mass of dust and mass of pure ice grains derived from the dense medium model, and corresponding dust-to-ice ratios.

\begin{tabular}{lccccc}
\hline \hline $\begin{array}{l}\text { Size range } \\
(\mu \mathrm{m})\end{array}$ & & $(1)$ & \multicolumn{4}{c}{ Parameter set } \\
& & $(2)$ & $(3)$ & $(4)$ \\
\hline$\leq 1$ & $M_{\text {ice }}(\mathrm{kg})$ & $\approx 7.6 \times 10^{6}$ & $\approx 1.6 \times 10^{9}$ & $\approx 7.6 \times 10^{6}$ & $\approx 1.8 \times 10^{7}$ \\
& $M_{\mathrm{d}}^{\text {tot }}(\mathrm{kg})$ & $1.2 \times 10^{5}$ & $8.1 \times 10^{4}$ & $1.2 \times 10^{5}$ & $6.6 \times 10^{4}$ \\
& $M_{\mathrm{d}}^{\text {tot }} / M_{\text {ice }}$ & $\approx 0.02$ & $\approx 5 \times 10^{-5}$ & $\approx 0.02$ & $\approx 0.004$ \\
$\leq 10$ & $M_{\text {ice }}(\mathrm{kg})$ & $\approx 1.6 \times 10^{7}$ & $\approx 1.5 \times 10^{10}$ & $\approx 1.6 \times 10^{7}$ & $\approx 2.3 \times 10^{8}$ \\
& $M_{\mathrm{d}}^{\text {tot }}(\mathrm{kg})$ & $2.9 \times 10^{5}$ & $8.0 \times 10^{5}$ & $2.7 \times 10^{5}$ & $9.0 \times 10^{5}$ \\
& $M_{\mathrm{d}}^{\text {tot }} / M_{\text {ice }}$ & $\approx 0.02$ & $\approx 5 \times 10^{-5}$ & $\approx 0.02$ & $\approx 0.004$ \\
$\leq 100$ & $M_{\text {ice }}(\mathrm{kg})$ & $\approx 2.3 \times 10^{7}$ & $\approx 4.7 \times 10^{10}$ & $\approx 2.3 \times 10^{7}$ & $\approx 5.4 \times 10^{8}$ \\
& $M_{\mathrm{d}}^{\text {tot }}(\mathrm{kg})$ & $5.6 \times 10^{5}$ & $3.6 \times 10^{6}$ & $4.7 \times 10^{5}$ & $2.2 \times 10^{6}$ \\
& $M_{\mathrm{d}}^{\text {tot }} / M_{\text {ice }}$ & $\approx 0.03$ & $\approx 8 \times 10^{-5}$ & $\approx 0.02$ & $\approx 0.004$ \\
\hline
\end{tabular}

Notes. The different sets of parameters are summarized in Table 5.

\section{Summary}

Using a time series of mid-infrared spectral maps from Spitzer obtained on 2005 July 04, we have analyzed the spatial and temporal evolution of water and dust and the thermal properties of dust grains ejected into the coma of comet 9P/Tempel 1 during the Deep impact collision.

Fitting the underlying continuum with a blackbody function, we have determined the color temperature of the dust. Before impact the color temperature was $265 \pm 2 \mathrm{~K}$. The color temperature of the ejecta was $375 \pm 5 \mathrm{~K}$ just after impact. We analyzed the grain properties of the ejecta using a dust thermal model for the continuum for two size distributions and grain compositions and found that a significant number of small grains were released as a result of impact and grain fragmentation. The maximum mass of dust in the Spitzer extraction aperture FOV $\left(9.25^{\prime \prime} \times 9.25^{\prime \prime}\right.$ centered on the nucleus) is obtained at TI $+3.6 \mathrm{~h}$, after which the mass decreases with time as small particles leave the FOV. The measured mass of submicron grains decreases faster than the mass of the larger grains because the population of the smallest grains in the inner coma decreases more quickly than the larger grains because the smaller grains are moving faster. The total mass of the ejecta ranges from $(2.7-8.9) \times 10^{5} \mathrm{~kg}$ for sizes $0.1-10 \mu \mathrm{m}$, and from $(0.5-2.1) \times 10^{6} \mathrm{~kg}$ for sizes $0.1-100 \mu \mathrm{m}$.

With a time dependent dust evolution model we fitted synthetic SEDs and derived dust parameters including the velocity distribution for the grain size distribution, for both the power law distribution and the Hanner distribution. We determined the velocity of the smallest grains, equal to $230 \mathrm{~m} \mathrm{~s}^{-1}$, from the observed Spitzer SED assuming that they leave the FOV at TI $+3.6 \mathrm{~h}$. Our model reasonably fits the observed flux until $\mathrm{TI}+10.2 \mathrm{~h}$; however, the flux observed at TI $+20.5 \mathrm{~h}$ is underestimated. The synthetic spectra obtained with the time dependent model for the dust also enabled an investigation of the temporal evolution of parameters for both size distributions. At TI $+20.5 \mathrm{~h}, a_{\mathrm{p}}$ is greater for the model than for the observations in the case of the Hanner distribution and $q$ is smaller for the model than for the observations in the case of the power law distribution. This behavior can be explained by the presence of an additional sources of small grains, i.e., the fragmentation of large grains, which is not taken into account in our model.

The water production rate determined from the spatial distribution of water within the coma before impact is $Q_{\text {pre }}=(4.70 \pm 0.7) \times 10^{27} \mathrm{~mol} \mathrm{~s}^{-1}$ assuming a spherical expansion of water with $v_{\text {exp }}=0.75 \mathrm{~km} \mathrm{~s}^{-1}$.

With a time-dependent model we studied the temporal evolution of the water molecules in the FOV after impact. This temporal evolution strongly suggests that sustained production of water molecules occured after impact from sublimating icy grains. We have found that a linear combination of a short-duration outburst and a long-duration outburst is needed with a relative proportion of water molecules injected during the short-duration outburst of 0.13 . The short-duration outburst $\left(t_{\text {burst }}=500 \mathrm{~s}\right)$ reproduces the strong production of water observed just after impact and a long-duration outburst is required $\left(t_{\text {burst }}=70000 \mathrm{~s}\right)$ to explain the sustained production of water after TI $+0.67 \mathrm{~h}$. We estimated the number of water molecules injected by the impact $N_{\text {inj }}=(2.5 \pm 0.5) \times 10^{32}$ molecules which corresponds to a mass of water $M_{\mathrm{inj}}=(7.4 \pm 1.5) \times 10^{6} \mathrm{~kg}$.

We showed that the sublimation of pure water ice grains (with sizes ranging from $0.1-0.6 \mu \mathrm{m}$ ) reproduces the overall temporal evolution at $\mathrm{TI}+1.83 \mathrm{~h}$ and later. We estimated the mass of ice ejected by the impact to $M_{\text {ice }}=(0.5-1.8) \times 10^{7} \mathrm{~kg}$, considering grain sizes $<1 \mu \mathrm{m}$. The ice-to-dust ratio is $\approx 30$ for the population of grains with sizes $<1 \mu \mathrm{m}$. The assumption of similar size distributions for dust and ice grains results in a high ice-to-dust ratio of $\approx 50$, assuming that dust and ice grains $<10-100 \mu \mathrm{m}$ populate the ejecta cloud. Concluding that such a large amount of ice is present below the surface seems unrealistic. An ice-to-dust ratio of $\approx 10$ is obtained by supposing a grain size cut-off for icy grains of $1 \mu \mathrm{m}$ and dust grains with sizes ranging up to $100 \mu \mathrm{m}$, which implies an enrichment factor of $\approx 6$ enrichment in subsurface ice.

An alternate explanation to the observed long-term production of water is that water vapor was instead produced from the ejecta blanket formed by slow-moving pure and dirty ice grains falling back to the surface. If a large amount of material returned to the surface after the impact and sublimated, then the measured water/dust ratio in the ejecta cloud does not exclude an ice-todust ratio $\sim 1-3$ in the excavated material. A better understanding of the cratering event on $9 \mathrm{P} /$ Tempel 1 , and of the size distribution of icy grains, is required to provide stronger constraints on the ice-to-dust ratio in 9P/Tempel 1.

Acknowledgements. We thank D. Harker for enlightening discussions, and E. Lellouch for providing us the Mie code. This work is based on observations made with the Spitzer Space Telescope, which is operated by the Jet Propulsion Laboratory, California Institute of Technology under a contract with NASA. This research made use of Tiny Tim/Spitzer, developed by John Krist for the Spitzer Science Center. The Center is managed by the California Institute of Technology under a contract with NASA. C.E. Woodward also acknowledges support from National Science Foundation grant AST-0706980. M.S. Kelley acknowledges support from NASA Planetary Atmospheres grant NNX09AW16A. D.H. Wooden acknowledges support from NASA Planetary Atmospheres grant 08-PATM08-0080. 


\section{References}

A'Hearn, M. F. 2008, Space Sci. Rev., 138, 237

A'Hearn, M. F., Belton, M. J. S., Delamere, W. A., et al. 2005, Science, 310, 258

Beer, E. H., Podolak, M., \& Prialnik, D. 2006, Icarus, 180, 473

Biver, N., Bockelée-Morvan, D., Boissier, J., et al. 2007, Icarus, 187, 253

Bockelée-Morvan, D., \& Crovisier, J. 1989, A\&A, 216, 278

Bockelée-Morvan, D., Woodward, C. E., Kelley, M. S., \& Wooden, D. H. 2009, ApJ, 696, 1075

Bohren, C. F., \& Huffman, D. R. 1983, Absorption and scattering of light by small particles (New York: Wiley-Interscience)

Brownlee, D. E., Joswiak, D., Matrajt, G., \& Tsou, P. 2009, in Cosmic Dust,

Near and Far, ed. T. Henning, E. Grün, \& J. Steinacker, ASP Conf. Ser., 414, 157

Crifo, J. F., \& Rodionov, A. V. 1997, Icarus, 127, 319

Crovisier, J. 1989, A\&A, 213, 459

Davidsson, B. J. R., Gutiérrez, P. J., \& Rickman, H. 2009, Icarus, 201, 335

Delsemme, A. H., \& Miller, D. C. 1971, Planet. Space Sci., 19, 1229

DiSanti, M. A., Villanueva, G. L., Bonev, B. P., et al. 2007, Icarus, 187, 240

Dorschner, J., Begemann, B., Henning, T., Jaeger, C., \& Mutschke, H. 1995, A\&A, 300, 503

Feaga, L. M., A'Hearn, M. F., Sunshine, J. M., Groussin, O., \& Farnham, T. L. 2007, Icarus, 190, 345

Feldman, P. D., Stern, S. A., Steffl, A. J., et al. 2007, Icarus, 187, 104

Fernández, Y. R., Lisse, C. M., Kelley, M. S., et al. 2007, Icarus, 187, 220

Gehrz, R. D., Roellig, T. L., Werner, M. W., et al. 2007, Rev. Sci. Inst., 78, 011302

Greenberg, J. M., \& Hage, J. I. 1990, ApJ, 361, 260

Groussin, O., A'Hearn, M. F., Li, J., et al. 2007, Icarus, 191, 63

Groussin, O., A'Hearn, M., Belton, M. J. S., et al. 2010, Icarus, 205, 627

Gunnarsson, M. 2003, A\&A, 398, 353

Hanner, M. S. 1984, Adv. Space Res., 4, 189

Hanner, M. S., \& Zolensky, M. E. 2010, in Lecture Notes in Physics 815, ed. T. Henning (Berlin: Springer Verlag), 203

Hanner, M. S., Tedesco, E., Tokunaga, A. T., et al. 1985, Icarus, 64, 11

Hanner, M. S., Lynch, D. K., \& Russell, R. W. 1994, ApJ, 425, 274

Hanner, M. S., Lynch, D. K., Russell, R. W., et al. 1996, Icarus, 124, 344

Harker, D. E., Wooden, D. H., Woodward, C. E., \& Lisse, C. M. 2002, ApJ, 580, 579
Harker, D. E., Woodward, C. E., \& Wooden, D. H. 2005, Science, 310, 278

Harker, D. E., Woodward, C. E., Wooden, D. H., Fisher, R. S., \& Trujillo, C. A. 2007, Icarus, 190, 432

Harris, A. W. 1998, Icarus, 131, 291

Holsapple, K. A., \& Housen, K. R. 2007, Icarus, 187, 345

Houck, J. R., Roellig, T. L., van Cleve, J., et al. 2004, ApJS, 154, 18

Ipatov, S. I., \& A'Hearn, M. F. 2011, MNRAS, 414, 76

Jorda, L., Lamy, P., Faury, G., et al. 2007, Icarus, 187, 208

Keller, H. U., Jorda, L., Küppers, M., et al. 2005, Science, 310, 281

Keller, H. U., Küppers, M., Fornasier, S., et al. 2007, Icarus, 187, 87

Kelley, M. S., \& Wooden, D. H. 2009, Planet. Space Sci., 57, 1133

Knight, M. M., Walsh, K. J., A'Hearn, M. F., et al. 2007, Icarus, 187, 199

Kolokolova, L., Hanner, M. S., Levasseur-Regourd, A.-C., \& Gustafson, B. A. S. 2004, Comets II, ed. M. C. Festou, H. U. Keller, \& H. A. Weaver, 577

Küppers, M., Bertini, I., Fornasier, S., et al. 2005, Nature, 437, 987

Lamy, P. L. 1974, A\&A, 35, 197

Lichtenegger, H. I. M., \& Komle, N. I. 1991, Icarus, 90, 319

Lien, D. J. 1990, ApJ, 355, 680

Lisse, C. M., A'Hearn, M. F., Hauser, M. G., et al. 1998, ApJ, 496, 971

Lisse, C. M., A'Hearn, M. F., Groussin, O., et al. 2005, ApJ, 625, L139

Lisse, C. M., VanCleve, J., Adams, A. C., et al. 2006, Science, 313, 635

Lisse, C. M., Kraemer, K. E., Nuth, J. A., Li, A., \& Joswiak, D. 2007, Icarus, 187,69

Meech, K. J., Ageorges, N., A'Hearn, M. F., et al. 2005, Science, 310, 265

Richardson, J. E., Melosh, H. J., Lisse, C. M., \& Carcich, B. 2007, Icarus, 190, 357

Schleicher, D. G., Barnes, K. L., \& Baugh, N. F. 2006, AJ, 131, 1130

Smith, J. D. T., Armus, L., Dale, D. A., et al. 2007, PASP, 119, 1133

Sugita, S., Ootsubo, T., Kadono, T., et al. 2005, Science, 310, 274

Sunshine, J. M., A'Hearn, M. F., Groussin, O., et al. 2006, Science, 311, 1453

Sunshine, J. M., Groussin, O., Schultz, P. H., et al. 2007, Icarus, 190, 284

Van de Hulst, H. C. 1957, Light Scattering by Small Particles (Courier Dover Publications)

Warren, S. G. 1984, Appl. Opt., 23, 1206

Werner, M. W., Uchida, K. I., Sellgren, K., et al. 2004, ApJS, 154, 309

Woodward, C. E., Kelley, M. S., Bockelée-Morvan, D., \& Gehrz, R. D. 2007, ApJ, 671, 1065 